\title{
Light-entrained and brain-tuned circadian circuits regulate ILC3 and gut homeostasis
}

Cristina Godinho-Silva ${ }^{{ }^{*}}$, Rita G. Domingues ${ }^{1^{*}}$, Miguel Rendas ${ }^{1}$, Bruno Raposo ${ }^{1}$, Hélder Ribeiro ${ }^{1}$, Joaquim Alves da Silva ${ }^{1,2}$, Ana Vieira ${ }^{1}$, Rui M. Costa $^{1,3}$, Nuno L. Barbosa-Morais ${ }^{4}$, Tânia Carvalho ${ }^{4}$, Henrique VeigaFernandes ${ }^{1}$

${ }^{1}$ Champalimaud Research. Champalimaud Centre for the Unknown. Champalimaud Foundation. 1400-038 Lisboa, Portugal

${ }^{2}$ Champalimaud Clinical Centre. Champalimaud Centre for the Unknown. Champalimaud Foundation. 1400-038 Lisboa, Portugal

${ }^{3}$ Zuckerman Mind Brain Behavior Institute, Columbia University, NY 10027, USA

${ }^{4}$ Instituto de Medicina Molecular, Faculdade de Medicina de Lisboa, Av. Prof. Egas Moniz, Edifício Egas Moniz, 1649-028 Lisboa, Portugal

*These authors contributed equally to this work 
Group 3 innate lymphoid cells (ILC3) are major regulators of inflammation, infection, microbiota composition and metabolism ${ }^{1}$. ILC3 and neuronal cells were shown to interact at discrete mucosal locations to steer mucosal defence ${ }^{2,3}$. Nevertheless, whether neuroimmune circuits operate at an organismal level, integrating extrinsic environmental signals to orchestrate ILC3 responses remains elusive. Here we show that light-entrained and brain-tuned circadian circuits regulate enteric ILC3, intestinal homeostasis, gut defence and the host lipid metabolism. We found that enteric ILC3 display circadian expression of clock genes and ILC3-related transcription factors. ILC3-autonomous ablation of the circadian regulator Arntl led to disrupted gut ILC3 homeostasis, impaired epithelial reactivity, deregulated microbiome, increased susceptibility to bowel infection and disrupted lipid metabolism. Loss of ILC3-intrinsic Arnt/ shaped the gut postcode receptors of ILC3. Strikingly, light-dark cycles, feeding rhythms and microbial cues differentially regulated ILC3 clocks, with light signals as major entraining cues of ILC3. Accordingly, surgical- and genetically-induced deregulation of brain rhythmicity led to disrupted circadian ILC3 oscillations, deregulated microbiome and altered lipid metabolism. Our work reveals a circadian circuitry that translates environmental light cues into enteric ILC3, shaping intestinal health, metabolism and organismal homeostasis.

Group 3 innate lymphoid cells (ILC3) were shown to be part of discrete mucosal neuroimmune cell units ${ }^{2-5}$, raising the hypothesis that ILC3 may also integrate systemic neuroimmune circuits to regulate tissue integrity and organismic homeostasis. Circadian rhythms rely on local and systemic cues to coordinate mammalian physiology and are genetically encoded by molecular clocks that allow organisms to anticipate and adapt to extrinsic environmental changes ${ }^{6,7}$. The circadian clock machinery consists of an autoregulatory network of feedback loops primarily driven by 
the activators ARNTL and CLOCK and the repressors PER1-3 and CRY1-2, amongst others $^{6,7}$.

Analysis of intestinal ILC subsets and their bone marrow (BM) progenitors revealed that mature ILC3 express high levels of circadian clock genes (Fig.1a-C and Extended Data Fig.1a-d). Importantly, ILC3 displayed a circadian pattern of Per1 ${ }^{\text {Venus }}$ expression (Fig.1b) and transcriptional analysis of ILC3 revealed circadian expression of master clock regulators and ILC3-related transcription factors (Fig.1c). To explore if ILC3 are regulated in a circadian manner, we investigated whether intestinal ILC3 require intrinsic clock signals. Thus, we interfered with the expression of the master circadian activator Arntl. Arntfl mice were bred to $V a v 1^{\text {Cre }}$ mice, allowing for conditional deletion of Arntl in all hematopoietic cells $\left(\left.A r n t\right|^{\mu \operatorname{Vav1}}\right.$ mice). While $\left.A r n t\right|^{\mu \text { Vav1 }}$ mice displayed normal numbers of intestinal NK cells and enteric groups 1 and 2 ILC, gut ILC3 were severely and selectively reduced when compared to their wild type littermate controls (Fig.1d,e and Extended Data Fig.2a,b). To more precisely define ILC3-intrinsic effects, we performed mixed BM chimeras transferring Arntl competent (Arntfl) or deficient $\left(\left.A r n t\right|^{\Delta \operatorname{Vav} 1}\right) B M$ against a third-part wild type competitor into alymphoid hosts (Fig.1f). Analysis of such chimeras confirmed a cell-autonomous circadian regulation of ILC3, while their innate and adaptive counterparts were unperturbed (Fig.1g and Extended Data Fig.2c).

To explore the functional impact of ILC3-intrisic circadian signals, we deleted Arntl in ROR $\gamma$ t expressing cells by breeding Rorgt $t^{\text {Cre }}$ to Arntfl mice (Arnt $\left.\right|^{\text {Rorgt }}$ mice). When compared to their wild type littermate controls, Arnt ${ }^{\Delta \text { Rorgt }}$ mice revealed selective reduction of ILC3 subsets and IL-17- and IL-22-producing ILC3 (Fig.2a,b and Extended Data Fig.3a-j). Noteworthy, independent deletion of Nr1d1 also perturbed enteric ILC3 subsets, further supporting a role of the clock machinery in ILC3 (Extended Data Fig.4a-e). ILC3 were shown to regulate epithelial reactivity gene expression and 
microbial composition ${ }^{1}$. Analysis of $A r n t f^{f l}$ and $\left.A r n t\right|^{\text {Rorgt }}$ mice revealed a profound reduction of reactivity genes in the $A r n t^{\Lambda^{\text {Rorgt }}}$ intestinal epithelium; notably, Reg3b, Reg3g, Muc3 and Muc13 were consistently reduced in Arntl deficient mice (Fig.2c). Furthermore, Arnt ${ }^{\wedge \text { Rorgt }}$ mice displayed altered diurnal patterns of Proteobacteria and Bacteroidetes (Fig.2d and Extended Data Fig.3j). To interrogate whether disruption of ILC3-intrinsic ARNTL impacted enteric defence, we tested how Arnt/ ${ }^{\text {Rorgt }}$ mice responded to intestinal infection. To this end, Arnt $\left.\right|^{\text {Rorgt }}$ mice were bred to Rag $1^{-1-}$ mice to exclude putative T cell effects (Extended Data Fig.3g-i). Rag $1^{-{ }^{-\alpha}}$. Arnt ${ }^{{ }^{\text {Rorgt }}}$ mice were infected with the attaching and effacing bacteria Citrobacter rodentium ${ }^{2}$. When

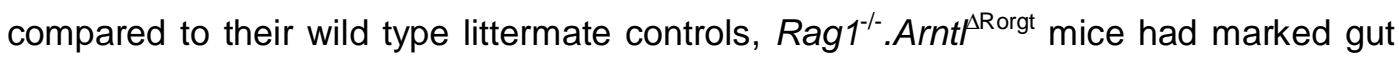
inflammation, reduced IL-22 producing ILC3, increased $C$. rodentium infection and bacterial translocation, reduced epithelial reactivity genes, increased weight loss and reduced survival (Fig.2e-j and Extended Data Fig.5a-j). These results indicate that cellintrinsic circadian signals selectively control intestinal ILC3 and shape gut epithelial reactivity, microbial communities and enteric defence. Previous studies indicated that ILC3 regulate host lipid metabolism ${ }^{8}$. When compared to their wild type littermate controls, the $\left.A r n t\right|^{\Delta \text { Rorgt }}$ epithelium revealed a marked increase in mRNA coding for key lipid epithelial transporters, including Fabp1, Fabp2, Scd1, Cd36 and Apoe (Fig.2k). Accordingly, these changes associated with increased gonadal and subcutaneous fat accumulation in $A r n t{ }^{\Lambda^{\text {Rorgt }}}$ mice when compared to their wild type littermate controls (Fig.2l and Extended Data Fig.5k-n). Thus, ILC3-intrinsic circadian signals shape epithelial lipid transport and body fat composition.

To further examine how cell-intrinsic Arntl controls intestinal ILC3 homeostasis, initially we interrogated the diurnal oscillations of the ILC3 clock machinery. When compared to their wild type littermate controls, Arnt/ ${ }^{\text {Rorgt }}$ ILC3 displayed a disrupted diurnal pattern of activator and repressor circadian genes (Fig.3a). Sequentially, we employed genome-wide transcriptional profiling of Arnt/ sufficient and deficient ILC3 to interrogate 
the impact of a deregulated circadian machinery. Diurnal analysis of the genetic signature associated with ILC3 identity ${ }^{1}$ demonstrated that the vast majority of those genes were unperturbed in Arntl deficient ILC3, suggesting that ARNTL is dispensable to ILC3-lineage commitment (Fig.3b and Extended Data Fig.6a-c). To test this hypothesis, we first interrogated the impact of Arntl ablation in ILC3 progenitors. Arnt ${ }^{\mathrm{V} \text { av1 }}$ mice had unperturbed numbers of common lymphoid progenitors (CLP) and innate lymphoid cell progenitors (ILCP) (Fig.3c and Extended Data Fig.6d). Sequentially, we analysed the impact of Arntl ablation in ILC3 residing in other organs. Compared to their littermate controls, $\left.A r n t\right|^{\wedge \text { Rorgt }}$ mice revealed normal numbers of ILC3 in the spleen, lung and blood, which was in large contrast to their pronounced reduction in the intestine (Fig.2a; Fig.3d,e and Extended Data 6e). Noteworthy, enteric Arnt/ ${ }^{\text {Rorgt }}$ ILC3 displayed unperturbed proliferation and apoptosis-related genetic signatures (Extended Data Fig.6b,c), suggesting that Arnt $A^{\text {Rorgt }}$ ILC3 may have altered intestinal traffic $^{9}$. When compared to their wild type littermate controls, Arnt/ ${ }^{\text {Rorgt }}$ ILC3 had a marked reduction of essential receptors for intestinal lamina propria homing and accumulated in mesenteric lymph-nodes (Extended Data Fig.6f) ${ }^{9}$. Notably, the integrin and chemokine receptors CCR9, $\alpha 4 \beta 7$ and CXCR 4 were selectively and hierarchically affected in Arnt/ ${ }^{\text {Rorgt }}$ ILC3 (Fig.3f-h and Extended Data Fig.6g-m). To examine whether ARNTL could directly regulate Ccr9 expression we performed chromatin immunoprecipitation. Binding of ARNTL to the Ccr9 locus of ILC3 followed a diurnal pattern, with increased binding at ZT5 (Fig.3i). Thus, ARNTL can directly contribute to Ccr9 expression in ILC3, although additional factors may also regulate this gene. In conclusion, while a fully operational ILC3-intrinsic circadian machinery is dispensable to ILC3-lineage commitment and development, cell-intrinsic clock signals are required for a functional ILC3 gut receptor postcode.

Circadian rhythms allow organisms to adapt to extrinsic environmental changes. Microbial cues were shown to impact the rhythms of intestinal cells ${ }^{10,11}$, while feeding 
regimens are major circadian entraining cues of peripheral organs, such as the liver ${ }^{12}$. In order to define the environmental cues that entrain circadian oscillations of ILC3, initially we investigated whether microbial cues impact the oscillations of ILC3. Per1 ${ }^{\text {Venus }}$ reporter mice treated with antibiotics displayed an unperturbed circadian oscillatory amplitude, while exhibiting a minute shift of their acrophase (Fig.4a). Sequentially, we tested whether feeding regimens, known to be major entraining cues of liver, pancreas, kidney, and heart oscillations ${ }^{12}$, impact ILC3 rhythms. To this end, we restricted food access to a 12 hours interval and compared Per $1^{\text {Venus }}$ oscillations to those observed in mice with inverted feeding regimens ${ }^{12}$. Inverted feeding had a small impact in the amplitude of ILC3 oscillations but did not invert the acrophase of ILC3 (Fig.4b and Extended Data Fig.7a), which was in large contrast with the full inversion of the acrophase of hepatocytes (Extended Data Fig.7b) ${ }^{12}$. Since these local intestinal cues could not invert the acrophase of ILC3, we hypothesise that light-dark cycles are major regulators of enteric ILC3 oscillations ${ }^{6}$. To test this hypothesis, we placed Per1 $1^{\text {Venus }}$ mice in light-tight cabinets on two opposing 12 hours light-dark cycles. Strikingly, inversion of light-dark cycles had a profound impact in the circadian oscillations of ILC3 (Fig.4c). Notably, and in contrast to microbiota and feeding regimens, light cycles fully inverted the acrophase of Per1 ${ }^{\text {Venus }}$ oscillations in ILC3 (Fig.4c and Extended Data Fig.7c). Furthermore, light-dark cycles entrained ILC3 oscillations as revealed by their maintenance upon removal of light (constant darkness) (Fig.4d and Extended Data Fig.7d), formally supporting that light is a major environmental entraining signal of ILC3 intrinsic oscillations. Together, these data indicate that ILC3 integrate systemic and local cues hierarchically; while microbiota and feeding regimens locally adjust the ILC3 clock, light-dark cycles are major entraining cues of ILC3, fully setting and entraining their intrinsic oscillatory clock.

The suprachiasmatic nuclei $(\mathrm{SCN})$ in the hypothalamus are main integrators of light signals ${ }^{6}$, suggesting that brain cues may regulate ILC3. To assess the impact of the 
master circadian pacemaker in ILC3, while excluding confounding light-induced, SCNindependent effects ${ }^{13,14}$, we performed SCN ablation by electrolytic lesion in Per1 $1^{\text {venus }}$ mice using stereotaxic brain surgery ${ }^{15}$. Strikingly, while sham operated mice displayed circadian Per1 ${ }^{\text {Venus }}$ oscillation in ILC3, their counterparts from SCN ablated mice fully lost the circadian rhythmicity of Per1 ${ }^{\text {Venus }}$ and other circadian genes (Fig.4e,f and Extended Data Fig.8a-d). Since electrolytic lesions of the SCN may cause scission of afferent and efferent fibres in the $\mathrm{SCN}$, we further confirmed that brain SCN-derived cues control ILC3 by the genetic ablation of Arntl in the $\mathrm{SCN}^{14}$. Arntf $f^{f l}$ mice were bred to Camk2a $a^{\text {Cre }}$ mice allowing for a forebrain/SCN-specific deletion of Arntl $\left(\text { Arnt }^{\text {ACamk2a }^{14}}\right)^{14}$. When compared to their control counterparts, ILC3 from Arnt ${ }^{\wedge \text { Camk2a }}$ mice revealed severe arrhythmicity of circadian regulatory genes and of the enteric postcode molecule CCR9 (Fig.4g,h and Extended Data Fig.9a-f). Importantly, Arnt/ ${ }^{\mathrm{Camk2a}}$ mice further exhibited altered epithelial reactivity genes and perturbed microbial communities, notably Proteobacteria and Bacteroidetes (Fig.4i,j and Extended Data Fig.9g-i). Finally, the Arnt ${ }^{\text {Camk2a }}$ intestinal epithelium showed disrupted circadian expression of lipid epithelial transporters, and these changes associated with increased gonadal and subcutaneous fat accumulation (Fig. 4k,l). Taken together, these data indicate that light-entrained and brain-tuned circuits regulate enteric ILC3, controlling microbial communities, lipid metabolism and body composition.

Deciphering the mechanisms by which neuroimmune circuits operate to integrate extrinsic and systemic signals is critical to understand tissue and organ homeostasis. We found that light cues are major extrinsic entraining cues of ILC3 circadian rhythms and surgical- and genetically-induced deregulation of brain rhythmicity resulted in altered ILC3 regulation. In turn, the ILC3-intrinsic circadian machinery controlled the gut receptor postcode of ILC3, shaping enteric ILC3 and host homeostasis. 
Our data reveal that ILC3 display diurnal oscillations that are genetically encoded, cellautonomous and entrained by light cues. While microbiota and feeding regimens could locally induce small adjustments to ILC3 oscillations, light-dark cycles were major entraining cues of the ILC3 circadian clock. Whether the effects of photonic signals on ILC3 are immediate or rely on other peripheral clocks remains to be elucidated ${ }^{16,17}$. Nevertheless, cell-intrinsic ablation of important endocrine and peripheral neural signals in ILC3 did not affect gut ILC3 numbers (Extended Data Fig.10a-i). Our work indicates that ILC3 integrate local and systemic entraining cues in a distinct hierarchical manner, establishing an organismal circuitry that is an essential link between the extrinsic environment, enteric ILC3, gut defence, lipid metabolism and host homeostasis (Extended Data Fig.10j).

Previous studies demonstrated that ILC integrate tissue microenvironmental signals, including cytokines, micronutrients and neuroregulators $3,4,18,19$. Here we show that ILC3 have a cell-intrinsic circadian clock that integrates extrinsic light-entrained and braintuned signals. Coupling light cues to ILC3 circadian regulation may have ensured efficient and integrated multi-system anticipatory responses to environmental changes. Notably, the regulation of ILC3 activity by systemic circadian circuits may have been selected to maximize metabolic homeostasis, gut defence and efficient symbiosis with commensals that have been evolution partners of mammals. Finally, our current data may also contribute to a better understanding of how circadian disruptions in humans associate with metabolic diseases, bowel inflammatory conditions and cancer ${ }^{20}$. 


\section{References}

1 Vivier, E. et al. Innate Lymphoid Cells: 10 Years On. Cell 174, 1054-1066 (2018).

2 Ibiza, S. et al. Glial-cell-derived neuroregulators control type 3 innate lymphoid cells and gut defence. Nature 535, 440-443 (2016).

3 Veiga-Fernandes, H. \& Artis, D. Neuronal-immune system cross-talk in homeostasis. Science 359, 1465-1466 (2018).

4 Godinho-Silva, C., Cardoso, F. \& Veiga-Fernandes, H. Neuro-Immune Cell Units: A New Paradigm in Physiology. Annu Rev Immunol (2018).

5 Veiga-Fernandes, H. \& Pachnis, V. Neuroimmune regulation during intestinal development and homeostasis. Nat Immunol 18, 116-122 (2017).

6 Takahashi, J. S. Transcriptional architecture of the mammalian circadian clock. Nat Rev Genet 18, 164-179 (2017).

7 Scheiermann, C., Gibbs, J., Ince, L. \& Loudon, A. Clocking in to immunity. Nat Rev Immunol 18, 423-437 (2018).

8 Mao, K. et al. Innate and adaptive lymphocytes sequentially shape the gut microbiota and lipid metabolism. Nature 554, 255-259 (2018).

9 Mackley, E. C. et al. CCR7-dependent trafficking of RORgamma(+) ILCs creates a unique microenvironment within mucosal draining lymph nodes. Nat Commun 6, 5862 (2015).

10 Thaiss, C. A. et al. Microbiota Diurnal Rhythmicity Programs Host Transcriptome Oscillations. Cell 167, 1495-1510 e1412 (2016).

11 Wang, Y. et al. The intestinal microbiota regulates body composition through NFIL3 and the circadian clock. Science 357, 912-916 (2017).

12 Damiola, F. et al. Restricted feeding uncouples circadian oscillators in peripheral tissues from the central pacemaker in the suprachiasmatic nucleus. Genes \& development 14, 2950-2961 (2000).

13 Husse, J., Leliavski, A., Tsang, A. H., Oster, H. \& Eichele, G. The light-dark cycle controls peripheral rhythmicity in mice with a genetically ablated suprachiasmatic nucleus clock. FASEB J 28, 4950-4960 (2014).

14 Izumo, M. et al. Differential effects of light and feeding on circadian organization of peripheral clocks in a forebrain Bmal1 mutant. eLife 3 (2014).

15 Sujino, M. et al. Suprachiasmatic nucleus grafts restore circadian behavioral rhythms of genetically arrhythmic mice. Current biology : $C B$ 13, 664-668 (2003).

16 Koronowski, K. B. et al. Defining the Independence of the Liver Circadian Clock. Cell 177, 1448-1462 e1414 (2019). 
17 Welz, P. S. et al. BMAL1-Driven Tissue Clocks Respond Independently to Light to Maintain Homeostasis. Cell 177, 1436-1447 e1412 (2019).

18 van de Pavert, S. A. et al. Maternal retinoids control type 3 innate lymphoid cells and set the offspring immunity. Nature 508, 123-127 (2014).

19 Cardoso, V. et al. Neuronal regulation of type 2 innate lymphoid cells via neuromedin U. Nature 549, 277-281 (2017).

20 Masri, S. \& Sassone-Corsi, P. The emerging link between cancer, metabolism, and circadian rhythms. Nat Med 24, 1795-1803 (2018). 
Figure 1. Intestinal ILC3 are controlled in a circadian manner. a, Common lymphoid progenitor (CLP); innate lymphoid cell progenitors (ILCP); intestinal ILC3. CLP and ILCP $n=4 ; \operatorname{ILC} 3 n=6$. b, Per1 ${ }^{\text {Venus }}$ MFI. CLP and ILCP $n=6$; ILC3 $n=4$. c, enteric ILC3. $n=5$. d, Intestinal ILC subsets. $n=4$. e, Gut ILC3. $n=4$. f, g, Mixed BM chimeras. Arntf $f^{i l}=5, A r n t / 1^{\Delta \operatorname{Vav} 1} \mathrm{n}=7$. (b-d) White/Grey: light/dark period. Data are representative of 3 independent experiments. $n$ represents biologically independent samples $(a, c)$ or animals (b,d-g). Mean and error bars: s.e.m. (a) two-way ANOVA and Tukey's test; (b,c) Cosinor analysis; (d,e,g) two-tailed Mann-Whitney $U$ test. ${ }^{*} \mathrm{P}<0.05$; ${ }^{* *} \mathrm{P}<0.01 ;{ }^{* * *} \mathrm{P}<0.001 ;$ ns not significant.

Figure 2. ILC3-intrinsic Arntl regulates gut homeostasis and defence. a, Enteric ILC3. $n=4$. b, Gut $T$ helper cells. $n=5$. c, Epithelial reactivity genes. $n=5 d$, Proteobacteria. Arnt $\left.\right|^{f l} \mathrm{n}=5 ;$ Arnt $\left.\right|^{\Delta \text { Rorgt }} \mathrm{n}=6$. e, Colon of $C$. rodentium infected mice. $\mathrm{n}=5$. f, Colitis score. $\mathrm{n}=5 . \mathbf{g}$, Colon length. $\mathrm{n}=5$. $\mathbf{h}$, Infection burden. $\operatorname{Rag}^{-1 /}$. Arnt $^{\mathrm{il}} \mathrm{n}=6, \operatorname{Rag}^{-}$

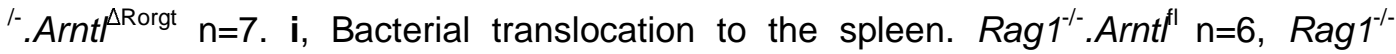
Arnt $^{\text {RRorgt }} \mathrm{n}=7$. j, Survival. $\mathrm{n}=5 ; \mathbf{k}$, Epithelial lipid transporter genes. $\operatorname{Arnt}^{\mathrm{fl}} \mathrm{n}=4$; Arnt ${ }^{\text {Rorgt }} \mathrm{n}=5$. I, Adipose tissue. $\mathrm{n}=5$. (d) White/Grey: light/dark period. Scale bars: $250 \mu \mathrm{m}$. Data are representative of at least 3 independent experiments. (a-l) $\mathrm{n}$ represents biologically independent animals. Mean and error bars: s.e.m. (a,b,f,g,i,k) two-tailed Mann-Whitney U test; (d) Cosinor analysis; (h) two-way ANOVA and Sidak's test; (j) Log-rank test; (I) two-tailed unpaired Student t-test. ${ }^{*} P<0.05$; ${ }^{* *} P<0.01$; ${ }^{* * *} \mathrm{P}<0.001$; ns not significant.

Figure 3. ILC3-intrinsic circadian signals regulate an enteric receptor postcode. a, Enteric ILC3. n=3. b, RNA-seq. Gut ILC3. n=3. c, CLP and ILCP. n=4. d, e, ILC3. (d) spleen, $n=3$; (e) lung $n=6$. $\mathbf{f}, \mathbf{g}$, Gut ILC3. $n=4$. h, intestinal ILC3. $n=4$. i, ChIP analysis in enteric ILC3. $n=3$. Data are representative of 3 independent experiments. $n$ represents biologically independent animals (a,c-h) or samples (b,i). (i) A-J putative ARNTL DNA binding sites. (a,h) White/Grey: light/dark period. Mean and error bars: 
s.e.m. (a) two-way ANOVA; (c-g) two-tailed Mann-Whitney U test; (h) Cosinor analysis; (i) two-tailed unpaired Student t-test. ${ }^{*} \mathrm{P}<0.05$; ${ }^{* *} \mathrm{P}<0.01$; ${ }^{* *} \mathrm{P}<0.001$; ns not significant.

Figure 4. Light-entrained and brain-tuned cues shape intestinal ILC3. a, Antibiotic treatment. Per1 ${ }^{\text {Venus }}$ in gut ILC3. $\mathrm{n}=3$. b, Restricted and inverted feeding. Per1 ${ }^{\text {Venus }}$ in gut ILC3. n=3. c, Opposing light-dark cycles. Per1 ${ }^{\text {Venus }}$ in gut ILC3. n=3. d, Opposing light-dark cycles followed by constant darkness. Per1 $1^{\text {Venus }}$ in gut ILC3. $\mathrm{n}=3$. e, Magnetic resonance imaging of sham and SCN ablated (xSCN) mice. $\mathrm{n}=11 . \mathbf{f}, \mathbf{g}$, Enteric ILC3. $n=3$. h, Gut ILC3. $n=3$. i, Epithelial reactivity genes. $n=3$. j, Proteobacteria. $n=4 . \mathbf{k}$, Epithelial lipid transporter genes. $n=3$. I, Adipose tissue. Arnt $f^{f l} n=5, \operatorname{Arnt}^{\Delta C^{C a m k 2 a}} \mathrm{n}=4$. (a,b) White/Grey: light/dark period. Mean and error bars: s.e.m.. (a-I) n represents biologically independent animals. (a-d;f-k) Cosinor analysis; (f-k) Cosine fitted curves; amplitude (Amp) and acrophase (Acro) were extracted from the Cosinor model. (I) twotailed unpaired Student t-test. ${ }^{*} \mathrm{P}<0.05$; ${ }^{* *} \mathrm{P}<0.01$; ${ }^{* \star *} \mathrm{P}<0.001$; ns not significant. 
Acknowledgements. We thank the Vivarium, Flow Cytometry, Histology, Molecular Biology and Hardware platforms at the Champalimaud Centre for the Unknown. We thank the Congento infrastructure for genetic model organisms. We thank Roksana Pirzgalka, Roel Klein Wolterink, Sara Correia, Inês Godinho, Filipa Cardoso, Bethania Garcia Cassani and Kristin Fischer for technical help and discussions; Catherine French for helping in behaviour analysis; Noam Shemesh, Teresa Serradas Duarte and Daniel Nunes for MRI imaging. Artur Silva for technical help with hardware; Filipa RijoFerreira and Leopoldo Petreanu for helpful discussions. Pedro Faísca for pathology scoring. C.G.-S., R.G.D and M.R were supported by Fundação para a Ciência e Tecnologia (FCT), Portugal. N.L.B.-M. is supported by FCT, Portugal, and European Molecular Biology Organisation (EMBO). H.V.-F. by ERC (647274), EU, The Paul G. Allen Frontiers Group, US, and FCT, Portugal.

Author contribution. C.G.-S. and R.G.D designed, performed and analysed the experiments in Fig.1-4; and Extended data Fig.1-10. M.R. performed circadian analysis. M.R. and H.R provided technical assistance in Fig.2d-j and Extended Data Fig.5. M.R. provided technical assistance in Fig.3b; Extended Data Fig.6b,e. B.R. provided technical assistance in Fig.1a-d; Fig.3a,h,i; Fig.4j-k; Extended Data Fig.1, Extended Data Fig.3f,j; Extended Data Fig.6a,k,I and Extended Data Fig.8d; Extended Data Fig.9e-h. H.R. managed the animal colony. J.A.S. and R.M.C. helped designing the experiments in Fig.4e and Extended Data Fig.8a. A.V. provided technical assistance with flow cytometry. N.L.B.-M. analysed the experiments in Fig.3b and Extended Data Fig.6b. T.C. analysed the experiments in Fig.2e,f and Extended Data Fig.5a-c and Extended Data Fig.8d. H.V.-F. supervised the work, planned the experiments and wrote the manuscript.

Author information. The authors declare no competing financial interests. Correspondence and requests for materials should be addressed to H.V.-F.: henrique.veigafernandes@research.fchampalimaud.org. 
Data availability. Source data for quantifications shown in all graphs plotted in figures and extended data figures are available in the online version of the paper. The data sets generated in this study are also available from the corresponding author upon reasonable request. RNA-seq datasets analysed are publicly available in Gene Expression Omnibus repository with the accession number GSE135235. 


\section{Methods}

Mice: Nod/Scid/Gamma (NSG) were purchased from the Jackson Laboratories. C57BL/6J Ly5.1 were purchased from the Jackson Laboratories and bred with C57BL/6J in order to obtain C57BL/6 Ly5.1/Ly5.2 (CD45.1/CD45.2). $\operatorname{Rag}^{-1 / 21}$, Rag2 ${ }^{/-}$

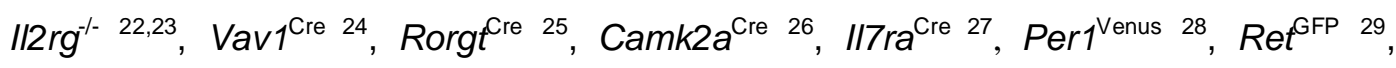

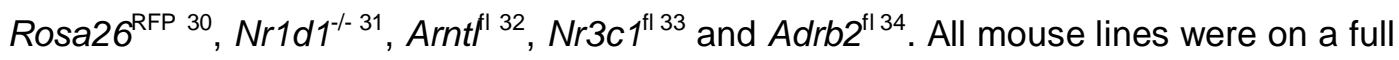
C57BL/6J background. All lines were bred and maintained at Champalimaud Centre for the Unknown (CCU) animal facility under specific pathogen free conditions. 8-14 weeks old males and females were used in this study, unless stated otherwise. Sex and age matched mice were used for small intestine epithelium lipid transporters analysis and white adipose tissue quantification. Mice were maintained in $12 \mathrm{~h}$ light-dark cycles, with ad libitum access to food and water, if not specified otherwise. For light inversion experiments mice were housed in ventilated, light-tight cabinets on defined $12 \mathrm{~h}$ lightdark cycles (Ternox). Camk2a $a^{\mathrm{Cr}} \cdot$ Arntf $^{f l}\left(\right.$ Arnt $\left.^{\wedge \mathrm{Camk2a}}\right)$ and their WT littermate controls were maintained in constant darkness as previously described ${ }^{14}$. Mice were systematically compared with co-housed littermate controls unless stated otherwise. Power analysis was performed to estimate the number of experimental mice. All animal experiments were approved by national and local Institutional Review Boards (IRB), respectively, Direção Geral de Veterinária and CCU ethical committees. Randomisation and blinding were not used unless stated otherwise.

Cell isolation: Isolation of small intestine and colonic lamina propria cells was previously described ${ }^{2}$. Briefly, intestines and colons were thoroughly rinsed with cold PBS1X, Peyer patches were removed from the small intestine, and intestines and colons were cut in $1 \mathrm{~cm}$ pieces, and shaken for $30 \mathrm{~min}$ in PBS containing $2 \%$ FBS, $1 \%$ HEPES and 5mM EDTA to remove intraepithelial and epithelial cells. Intestines and colons were then digested with collagenase $D(0.5 \mathrm{mg} / \mathrm{mL}$; Roche) and DNase I $\left(20 \mathrm{U} / \mathrm{mL}\right.$; Roche) in complete RPMI for $30 \mathrm{~min}$ at $37^{\circ} \mathrm{C}$, under gentle agitation. 
Sequentially cells were passed through a $100 \mu \mathrm{M}$ cell strainer and purified by centrifugation 30min at 2400rpm in 40/80 Percoll (GE Healthcare) gradient. Lungs were finely minced and digested in complete RPMI supplemented with collagenase D $\left(0.1 \mathrm{mg} / \mathrm{mL}\right.$; Roche) and DNase I $\left(20 \mathrm{U} / \mathrm{mL}\right.$; Roche) for $1 \mathrm{~h}$ at $37^{\circ} \mathrm{C}$ under gentle agitation. Sequentially, cells were passed through a $100 \mu \mathrm{M}$ cell strainer purified by centrifugation 30 minutes at 2400rpm in 40/80 Percoll (GE Healthcare) gradient. Spleen and mesenteric lymph node cell suspensions were obtained using $70 \mu \mathrm{m}$ strainers. Bone marrow cells were collected by either flushing or crushing bones and filtered using $70 \mu \mathrm{m}$ strainers. Erythrocytes from small intestine, colon, lung, spleen and bone marrow preparations were lysed with RBC lysis buffer (eBioscience). Leukocytes from blood were isolated by treatment with Ficoll (GE Healthcare).

Flow cytometry analysis and cell sorting: For cytokine analysis ex vivo, cells were incubated with PMA (phorbol 12-myristate 13-acetate; $50 \mathrm{ng} / \mathrm{mL}$ ) and ionomycin (500ng/mL) (Sigma-Aldrich) in the presence of brefeldin A (eBioscience) for 4 hours prior to intracellular staining. Intracellular staining for cytokines and transcription factors analysis was performed using IC fixation and Staining Buffer Set (eBioscience). Cell sorting was performed using FACSFusion (BD Biosciences). Sorted populations were $>95 \%$ pure. Flow cytometry analysis was performed on LSRFortessa X-20 (BD Biosciences). Data was analysed using FlowJo 8.8.7 software (Tree Star). Cell populations were gated in live cells, both for sorting and flow cytometry analysis.

Cell populations: Cell populations were defined as: Bone marrow (BM) Common

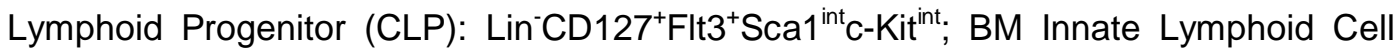

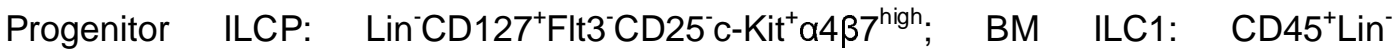

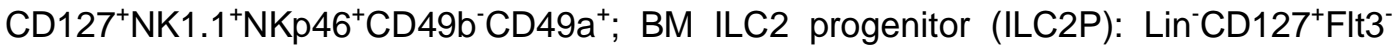
Sca $1^{+} \mathrm{CD} 25^{+}$; small intestine (SI) NK: CD45 ${ }^{+}$in NK1.1 ${ }^{+} \mathrm{NKp} 46^{+} \mathrm{CD} 27^{+} \mathrm{CD} 49 \mathrm{~b}^{+} \mathrm{CD} 127^{-}$ $\mathrm{EOMES}^{+}$or $\mathrm{CD}^{+} 5^{+} \mathrm{Lin}^{-} \mathrm{NK} 1.1^{+} \mathrm{NKp} 46^{+} \mathrm{CD} 27^{+} \mathrm{CD} 49 \mathrm{~b}^{+} \mathrm{CD} 127$; small intestine ILC1: 
NK1. $1^{+} \mathrm{NKp} 46^{+} \mathrm{CD} 27^{+} \mathrm{CD} 49 \mathrm{~b}{ }^{-C D} 127^{+}$; $\quad$ small intestine ILC2: ${\text { CD } 45^{+} \text {Lin }^{-}}^{-}$ Thy $1.2^{+} \mathrm{KLRG} 1^{+} \mathrm{GATA} 3^{+}$or $\mathrm{CD} 45^{+} \mathrm{Lin}{ }^{-T h y} 1.2^{+} \mathrm{KLRG} 1{ }^{+} \mathrm{Sca}-1^{+} \mathrm{CD} 25^{+}$; lamina propria, spleen, mesenteric lymph node and lung ILC3: CD45+Lin-Thy1.2 ${ }^{\text {high }}$ RORyt $^{+}$or

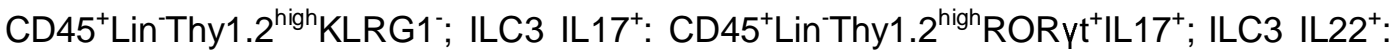
CD45 ${ }^{+}$Lin Thy1.2 ${ }^{\text {high }} \mathrm{RORyt}^{+} \mathrm{IL} 22^{+}$; for ILC3 subsets additional markers were employed:

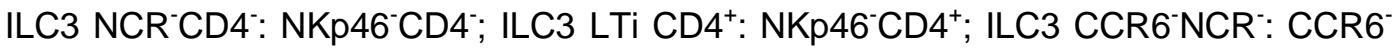
NKp46; ILC3 LTi-like: CCR6+NKp46; ILC3 NCR ${ }^{+}$: NKp46+; SI T helper (Th) 17 cells: CD45 ${ }^{+}$in $^{+}$Thy $1.2^{+} \mathrm{CD}^{+}{ }^{+}$OR t $^{+}$; colon Tregs: CD $45^{+} \mathrm{CD}^{+}{ }^{+}$Thy $1.2^{+} \mathrm{CD}^{+} \mathrm{CD}^{+} 5^{+} \mathrm{FOXP}^{+}$; colon Tregs RORyt ${ }^{+}$CD $45^{+} \mathrm{CD} 3^{+}$Thy $1.2^{+} \mathrm{CD} 4^{+} \mathrm{CD} 25^{+} \mathrm{FOXP} 3^{+} \mathrm{RORyt}^{+}$. The lineage cocktail for BM, lung, small intestine lamina propria, spleen and mesenteric lymph nodes included CD3 $\square$, CD8a, CD19, B220, CD11c, CD11b, Ter119, Gr1, TCR $\beta$, TCRyō and NK1.1. For NK and ILC1 staining in the small intestine NK1.1 and CD11b were not added to the lineage cocktail.

Antibody list: Cell suspensions were stained with: anti-CD45 (30-F11); anti-CD45.1 (A20); anti-CD45.2 (104); anti-CD11c (N418); anti-CD11b (Mi/70); anti-CD127 (IL7Ra; A7R34); anti-CD27(LG.7F9); anti-CD8a (53-6.7); anti-CD19 (eBio1D3); antiCXCR4(L276F12); anti-NK1.1 (PK136); anti-CD3 $\square$ (eBio500A2); anti-TER119 (TER119); anti-Gr1 (RB6-8C5); anti-CD4 (RM4-5); anti-CD25 (PC61); anti-CD117 (c-Kit; 2B8); anti-CD90.2 (Thy1.2; 53-2.1); anti-TCRß (H57-595); anti-TCRYס (GL3); anti-B220 (RA3-6B2); anti-KLRG1 (2F1/KLRG1); anti-Ly-6A/E (Sca1; D7); anti-CCR9 (CW-1.2); anti-IL-17 (TC11-18H10.1); anti-rat IgG1k isotype control (RTK2071); anti-streptavidin fluorochrome conjugates from Biolegend; anti-a4ß7 (DATK32); anti-Flt3 (A2F10); antiNKp46 (29A1.4); anti-CD49b (DX5); anti-Ki67 (SolA15); anti-rat IgG2ak isotype control (eBR2a); anti-IL-22 (1H8PWSR); anti-rat IgG1k isotype control (eBRG1); anti-EOMES (Dan11mag); anti-Tbet (eBio4B10); anti-FOPX3 (FJK-16s); anti-GATA3 (TWAJ); antiCD16/CD32 (93); 7AAD viability dye from eBiosciences; anti-CD196 (CCR6; 140706) from BD Biosciences; anti-ROR $\gamma$ (Q31-378) and anti-mouse lgG2ak isotype control 
(G155-178) from BD Pharmingen. LIVE/DEAD Fixable Aqua Dead Cell Stain Kit was purchased from Invitrogen.

Bone marrow transplantation: Bone marrow $\mathrm{CD}^{-}$cells were FACS sorted from

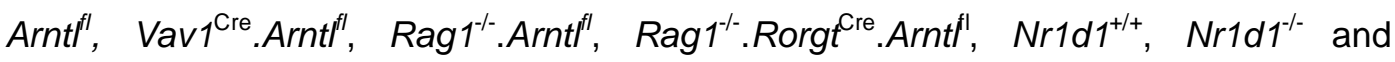
C57BL/6 Ly5.1/Ly5.2 mice. $2 \times 10^{5}$ sorted cells from Arntl deficient and competent wild type littermate controls, were intravenously injected in direct competition with a thirdpart wild type competitor (CD45.1/CD45.2), in a 1:1 ratio, into non-lethally irradiated NSG (150cGy) or Rag2/- II2rg ${ }^{-/-}$(500cGy) mice (CD45.1). Recipients were analysed at 8 weeks post-transplantation.

Quantitative RT-PCR: RNA from sorted cells was extracted using RNeasy micro kit (Qiagen) according to the manufacturer's protocol. Liver, small intestine (ileum) and colon epithelium was collected for RNA extraction using Trizol (Invitrogen) and zirconia/silica beads (BioSpec) in a bead beater (MIDSCI). RNA concentration was determined using Nanodrop Spectrophotometer (Nanodrop Technologies). For TaqMan assays (Applied Biosystems) RNA was retro-transcribed using a High Capacity RNA-to-cDNA Kit (Applied Biosystems), followed by a pre-amplification PCR using TaqMan PreAmp Master Mix (Applied Biosystems). TaqMan Gene Expression Master Mix (Applied Biosystems) was used in real-time PCR. Real time PCR analysis was performed using StepOne and QuantStudio 5 Real-Time PCR systems (Applied Biosystems). Hprt, Gapdh and Eef1a1 were used as housekeeping genes. When multiple endogenous controls were used, these were treated as a single population and the reference value calculated by arithmetic mean of their CT values. The mRNA analysis was performed as previously described ${ }^{35}$. Briefly, the comparative $\mathrm{C}_{\mathrm{T}}$ method $\left(2^{-\Delta \mathrm{CT}}\right.$ ) in which $\Delta \mathrm{C}_{\mathrm{T}}$ (gene of interest) $=\mathrm{C}_{\mathrm{T}}$ (gene of interest) $-\mathrm{C}_{\mathrm{T}}$ (Housekeeping reference value) was employed. When fold change comparison between samples was required, the comparative $\Delta \mathrm{CT}$ method $\left(2^{-\Delta \Delta \mathrm{CT}}\right)$ was applied. 
TaqMan Gene Expression Assays: TaqMan Gene Expression Assays (Applied Biosystems) were the following: Hprt Mm00446968_m1; Gapdh Mm99999915_g1; Eef1a1 Mm01973893_g1; Arntl Mm00500223_m1; Clock Mm00455950_m1; Nr1d1 Mm00520708_m; Nr1d2 Mm01310356_g1; Per1 Mm00501813_m1; Per2 00478113_m1; Cry1 Mm00500223_m1; Cry2 Mm01331539_m1; Runx1 Mm01213404_m1; Tox Mm00455231_m1; Rorgt Mm01261022_m1; Ahr Mm00478932_m1; Rora Mm01173766_m1; Ccr9 Mm02528165_s1; Reg3a Mm01181787_m1; Reg3b Mm00440616_g1; Reg3g Mm00441127_m1; Muc1 Mm00449604_m1; Muc2 Mm01276696_m1; Muc3 Mm01207064_m1; Muc13 Mm00495397_m1; S100a8 Mm01276696_m1; S100a9 Mm00656925_m1; Epcam Mm00493214_m1; Apoe Mm01307193_g1; Cd36 Mm01307193_g1; Fabp1 Mm00444340_m1; Fabp2 Mm00433188_m1; and Scd1 Mm00772290_m1.

Quantitative PCR analysis of bacteria in stools at the Phylum level: DNA from

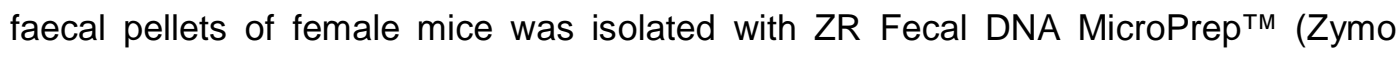
Research). Quantification of bacteria was determined from standard curves established by qPCR as previously described ${ }^{2}$. qPCRs were performed with NZY qPCR Green Master Mix (Nzytech) and different primer sets using a QuantStudio 5 Real-Time PCR System (Applied Biosystems) thermocycler. Samples were normalized to 165 rDNA and reported according to the $2^{-\triangle C T}$ method. Primer sequences were: $16 S$ rDNA, FACTCCTACGGGAGGCAGCAGT and R- ATTACCGCGGCTGCTGGC; Bacteroidetes, F- GAGAGGAAGGTCCCCCAC and R-CGCTACTTGGCTGGTTCAG; Proteobacteria, F- GGTTCTGAGAGGAGGTCCC and R- GCTGGCTCCCGTAGGAGT; Firmicutes, FGGAGCATGTGGTTTAATTCGAAGCA and R- AGCTGACGACAACCATGCAC.

Citrobacter rodentium infection: Infection with Citrobacter rodentium ICC180 (derived from DBS100 strain) ${ }^{36}$ was performed at ZT6 by gavage inoculation of $10^{9}$ colony forming units ${ }^{36,37}$. Acquisition and quantification of luciferase signal was 
performed in an IVIS Lumina III System (Perkin Elmer). Throughout infection, weight loss, diarrhoea and bloody stools were monitored daily.

Colony forming unit measurement: Bacterial translocation was determined in the spleen, liver, and mesenteric lymph nodes, taking in account total bacteria and Luciferase $^{\text {positive }}$ C. rodentium. Organs were harvested, weighted, and brought into suspension. Bacterial colony forming units (CFU) of organ samples were determined via serial dilutions on Luria Broth (LB) agar (Invitrogen) and MacConkey agar (SigmaAldrich). Colonies were counted after 2 days of culture at $37^{\circ} \mathrm{C}$. Luciferase ${ }^{\text {positive }} \mathrm{C}$. rodentium was quantified in MacConkey agar plates using an IVIS Lumina III System (Perkin Elmer). CFU were determined per volume $(\mathrm{mL})$ for each organ.

Antibiotic and dexamethasone treatment: Pregnant females and new born mice were treated with streptomycin 5g/L, ampicillin 1g/L and colistin 1g/L (Sigma-Aldrich) into drinking water with 3\% sucrose. Control mice were given 3\% sucrose in drinking water as previously described ${ }^{38}$. Dexamethasone 21 -phosphate disodium salt $(200 \mu \mathrm{g})$ (Sigma) or PBS was injected intraperitoneally at ZTO. After 4, 8, 12 and 23 hours (ZT 4, 8, 12 and 23) mice were sacrificed and analysed.

Chromatin immunoprecipitation (ChIP) assay: Enteric ILC3 from adult C57BL/6J mice were isolated by flow cytometry. Cells were fixed, cross-linked, lysed and chromosomal DNA-protein complex sonicated to generate DNA fragments ranging from 200-400 base pairs as previously described ${ }^{2}$. DNA-protein complexes were immunoprecipitated, using LowCell\# ChIP kit (Diagenode), with $1 \mu \mathrm{gg}$ of antibody against ARNTL (Abcam) and IgG isotype control (Abcam). Immunoprecipitates were uncrosslinked and analysed by quantitative PCR using primer pairs flanking ARNTL putative sites (E-boxes) in the Ccr9 locus (determined by computational analysis using TFBS tools and Jaspar 2018). Results were normalized to input intensity and control IgG. Primer sequences were: A: F-CATTTCATAGCTTAGGCTGGCATGG; R- 
CTAGCTAACTGGTCTCAAAGTCCTC; B: F-GCCTCCCTTGTACTACCTGAAGC; RTCCCAACACCAGGCCGAGTA; C: F-AGGGTCAATTTCTTAGGGCGACA; RGCCAAGTGTTCGGTCCCAC; D: F-TCTGGCTTCTCACCATGACCACT; RTCTAAGGCGTCACCACTGTTCTC, E: F-TTTGGGGAATCATCTTACAGCAGAG; RATTCATCCTGGCCCTTTCCTTCTTA; F: F-GCTCCACCTCATAGTTGTCTGG; RCCATGAGCACGTGGAGAGAAAG; $\quad$ G: F-GGTCGAATACCGCGTGGGTT; RCCCGGTAGAGGCTGCAAGAAA; H: F-AGGCAAATCTGGGCCTATCC; RGGCCCAGTACAGAGGGGTCT; I: F-GGCTCAGGCTAGCAGGTCTC; RTGTTTGGCCAGCATCCTCCA; J: F-ACTCAGAGGTGCTGTGACTCC; RAGCTTTAGGACCACAATGGGCA.

Food restriction (inverted feeding): $P e r 1^{\text {Venus }}$ mice fed during the night received food from 9pm to 9am (control group), whereas mice fed during the day had access to food from 9am to $9 \mathrm{pm}$ (inverted group). Food restriction was performed during 9 consecutive days as previously described ${ }^{12}$. For food restriction in constant darkness, Per1 ${ }^{\text {Venus }}$ mice were housed in constant darkness with ad libitum access to food and water for 2 weeks. Then, access to food was restricted to the subjective day or night, for 12 days, in constant darkness.

Inverted light-dark cycles: To induce changes in light regime, Per1 ${ }^{\text {Venus }}$ mice were placed in ventilated, light-tight cabinets on a $12 \mathrm{~h}$ light-dark cycle (Ternox). After acclimation, light cycles were changed for mice in the inverted group for 3 weeks to completely establish an inverse light cycle, while they remained the same for mice in the control group, as previously described ${ }^{39}$. For inverted light dark cycle experiments followed by constant darkness, after establishing an inverse light dark cycle, mice were transferred into constant darkness for 3 weeks.

SCN lesions: Bilateral ablation of the SCN was performed in 9 to 12 week-old Per1 $1^{\text {Venus }}$ males by electrolytic lesion using stereotaxic brain surgery, as described 
previously ${ }^{15}$. Mice were kept in deep anaesthesia using a mixture of isoflurane and oxygen (1-3\% isoflurane at $1 \mathrm{~L} / \mathrm{min})$. Surgeries were performed using a stereotaxic device (Kopf). After identification of bregma, a hole was drilled through which the lesion electrode was inserted into the brain. Electrodes were made by isolating a $0.25 \mathrm{~mm}$ stainless steel insect pin with a heat shrink polyester tubing, except for $0.2 \mathrm{~mm}$ at the tip. The electrode tip was aimed at the SCN, $0.3 \mathrm{~mm}$ anterior to bregma, $0.20 \mathrm{~mm}$ lateral to the midline, and $5.8 \mathrm{~mm}$ ventral to the surface of the cortex, according to the Paxinos Mouse Brain Atlas, 2001. Bilateral SCN lesions were made by passing a $1 \mathrm{~mA}$ current through the electrode for the duration of $6 \mathrm{sec}$, in the left and right SCN separately. Sham lesioned mice underwent the same procedure, but no current was passed through the electrode. After surgery animals were housed individually under constant dark conditions with ad libitum food and water and were allowed to recover for 1 week before behavioural analysis. Successful SCN lesioned mice were selected based on MRI imaging, arrhythmic behaviour and histopathology analysis.

Magnetic resonance imaging: Screening of SCN ablated mice was performed using a Bruker ICON scanner (Bruker, Karlsruhe, Germany). RARE (Rapid Acquisition with Refocused Echoes) sequence was used to acquire coronal, sagittal and axial slices (5 slices in each orientation) with the following parameters: RARE factor $=8, T E=85 \mathrm{~ms}$, $\mathrm{TR}=2500 \mathrm{~ms}$, resolution $=156 \times 156 \times 500 \mu \mathrm{m}^{3}$ (30 averages). For high-quality images a 9.4T BioSpec scanner (Bruker, Karlsruhe, Germany) was employed. This operates with Paravision 6.0.1 software and interfaced with an Avance IIIHD console. Anatomical images (16 axial and 13 sagittal slices) were acquired using a RARE (Rapid Acquisition with Refocused Echoes) sequence with RARE factor $=8, T E=36 \mathrm{~ms}, T R=2200 \mathrm{~ms}$ and resolution of $80 \times 80 \times 500 \mu \mathrm{m}^{3}$ (12 averages).

Behavioural analysis: Sham-operated and SCN ablated mice were individually housed and after a $24 \mathrm{~h}$ acclimation period their movement was recorded for $72 \mathrm{~h}$, starting at circadian time 7 , in constant darkness, using the automated animal 
behaviour CleverSys system. Data were auto scored by the CleverSys software. Videos and scoring were visually validated. Circadian rhythmicity was evaluated by cosinor regression model ${ }^{40,41}$.

Histopathology analysis: Mice infected with Citrobacter rodentium were sacrificed by $\mathrm{CO}_{2}$ narcosis, the gastrointestinal tract was isolated, and the full length of cecum and colon was collected and fixed in $10 \%$ neutral buffered formalin. Colon was trimmed in multiple transverse and cross-sections and cecum in one cross-section ${ }^{42}$, and all were processed for paraffin embedding. 3-4 $\mu \mathrm{m}$ sections were stained with haematoxylin and eosin and lesions were scored by a pathologist blinded to experimental groups, according previously published criteria ${ }^{43-45}$. Briefly, lesions were individually scored (04 increasing severity) for: 1-mucosal loss; 2-mucosal epithelial hyperplasia, 3-degree of inflammation, 4-extent of the section affected in any manner and 5-extent of the section affected in the most severe manner, as previously described ${ }^{45}$. Score was derived by summing the individual lesion and the extent scores. Mesenteric (mesocolic) inflammation was noted but not scored. Liver, gonadal and subcutaneous fat from Arnt $\left.\right|^{\text {Rorgt }}$ were collected, fixed in $10 \%$ neutral buffered formalin, processed for paraffin embedding, sectioned into $3 \mu \mathrm{m}$-thick sections and stained with haematoxylin and eosin. The presence of inflammatory infiltrates was analysed by a pathologist blinded to experimental groups. For the SCN lesions experiment, mice were sacrificed with $\mathrm{CO}_{2}$ narcosis, necropsy was performed, and brain was harvested and fixed in $4 \%$ PFA. Coronal section of $50 \mu \mathrm{m}$ thickness were performed in the vibratome (Leica VT1000 S), from 0.6 to -1.3 relatively to bregma, collected to Superfrost Plus slides (menzel-gläser) and let to dry overnight before Nissl staining. Stained slides were hydrated in distilled water for brief seconds and incubated in Cresyl Violet Stain solution (Sigma-Aldrich) for 30min. Slides were dehydrated in graded ethanol and mounted with CV Mount (Leica). Coronal sections were analysed for the presence/absence of SCN lesion (partial vs 
total ablation, unilateral vs bilateral), in a Leica DM200 microscope couple to a Leica MC170HD camera (Leica Microsystems, Wetzlar, Germany).

Microscopy: Adult intestines from $R e f^{\text {GFP }}$ mice were flushed with cold PBS (Gibco) and opened longitudinally. Mucus and epithelium were removed, intestines were fixed in $4 \%$ PFA (Sigma-Aldrich) at room temperature for 10 minutes and incubated in blocking/permeabilising buffer solution (PBS containing 2\% BSA, 2\% goat serum, $0.6 \%$ Triton X-100). Samples were cleared with benzyl alcohol-benzyl benzoate (SigmaAldrich) prior dehydration in methanol ${ }^{18,46}$. Whole-mount samples were incubated overnight or for 2 days at $4^{\circ} \mathrm{C}$ using the following antibodies: anti-Tyrosine hydroxylase (TH) (Pel-Freez Biologicals) and anti-GFP (Aves Labs). Alexa Fluor 488 goat antichicken and Alexa Fluor 568 goat anti-rabbit (Invitrogen) were used as secondary antibodies overnight at room temperature. For SCN imaging RFP ${ }^{\triangle C a m k 2 a}$ and RFP RRorgt $^{2}$ mice were anesthetized, perfused intracardially with PBS followed by $4 \%$ paraformaldehyde ( $\mathrm{pH} 7.4$, Sigma-Aldrich). The brains were removed and post-fixed for 24 hours in $4 \%$ paraformaldehyde and transferred to phosphate buffer. $50 \mu \mathrm{m}$ coronal sections were collected through the entire SCN using a Leica vibratome (VT1000s) into phosphate buffer and processed free-floating. Sections were incubated with neurotrace 500/525 (Invitrogen, N21480) diluted 1/200 and mounted using Mowiol. Samples were acquired on a Zeiss LSM710 confocal microscope using EC Plan-Neofluar 10x/0.30 M27, Plan Apochromat 20x/0.8 M27 and EC Plan-Neofluar 40x/1.30 objectives.

RNA sequencing and data analysis: RNA was extracted and purified from sorted small intestinal lamina propria cells isolated at ZT5 and ZT23. RNA quality was assessed by an Agilent 2100 Bioanalyzer. SMART-Seqll (ultra-low input RNA) libraries were prepared using Nextera XT DNA sample preparation kit (Illumina). Sequencing was performed on an Illumina HiSeq4000 platform, PE100. Global quality of FASTQ files with raw RNA-seq reads was analysed using fastqc (ver 0.11.5) (https://www.bioinformatics.babraham.ac.uk/projects/fastqc/). vast-tools ${ }^{47}$ (version 
2.0.0) aligning and read processing software was used for quantification of gene expression in read counts from FASTQ files using VASTD-DB ${ }^{47}$ transcript annotation for mouse genome assembly $\mathrm{mm} 9$. Only the 8443 genes with read count information in all 12 samples and an average greater than 1.25 reads/sample were considered informative enough for subsequent analyses. Preprocessing of read count data, namely transforming them to log2-counts per million (logCPM), was performed with $v{ }^{48}$, included in the Bioconductor ${ }^{49}$ package limma ${ }^{50}$ (version 3.38.3) for the statistical software environment $R$ (version 3.5.1). Linear models and empirical Bayes statistics were employed in differential gene expression analysis, using limma. For heatmaps, normalized RNAseq data was plotted using pheatmap (v1.0.10) R package (http://www.R-project.org/). Heatmap genes were clustered using Euclidean distance as metric. RNA-seq datasets analysed are publicly available in Gene Expression Omnibus repository with the accession number GSE135235.

Statistics: Results are shown as mean \pm s.e.m. Statistical analysis was performed using the GraphPad Prism software (version 6.01). Comparisons between two samples were performed using Mann-Whitney $U$ test or unpaired Student $t$ test. Two-way ANOVA analysis was used for multiple group comparisons, followed by Tukey post hoc test or Sidak's multiple comparisons test. Circadian rhythmicity was evaluated by Cosinor regression model ${ }^{40,41,51}$, using cosinor (v1.1) R package. Single-component Cosinor fits one cosine curve by least squares to the data. The circadian expression Period was assumed to be $24 \mathrm{~h}$ for all analysis and the significance of the circadian fit was assessed by a zero-amplitude test with $95 \%$ confidence. Single-component Cosinor yields estimates and defines standard errors with $95 \%$ confidence limits for Amplitude and Acrophase using Taylor's series expansion ${ }^{51}$. The latter were compared using two-tailed Student t-test, where indicated. Results were considered significant at ${ }^{*} \mathrm{P}<0.05,{ }^{* \star} \mathrm{P}<0.01,{ }^{* *} \mathrm{P}<0.001$. 
21 Mombaerts, $P$. et al. RAG-1-deficient mice have no mature $B$ and $T$ lymphocytes. Cell 68, 869-877. (1992).

22 Cao, X. et al. Defective lymphoid development in mice lacking expression of the common cytokine receptor gamma chain. Immunity 2, 223-238 (1995).

23 Shinkai, Y. et al. RAG-2-deficient mice lack mature lymphocytes owing to inability to initiate V(D)J rearrangement. Cell 68, 855-867. (1992).

24 de Boer, J. et al. Transgenic mice with hematopoietic and lymphoid specific expression of Cre. Eur J Immunol 33, 314-325 (2003).

25 Sawa, S. et al. Lineage relationship analysis of RORgammat+ innate lymphoid cells. Science 330, 665-669 (2010).

26 Casanova, E. et al. A CamKIlalpha iCre BAC allows brain-specific gene inactivation. Genesis 31, 37-42 (2001).

27 Schlenner, S. M. et al. Fate mapping reveals separate origins of $\mathrm{T}$ cells and myeloid lineages in the thymus. Immunity 32, 426-436 (2010).

28 Cheng, H. Y. et al. Segregation of expression of mPeriod gene homologs in neurons and glia: possible divergent roles of mPeriod1 and mPeriod2 in the brain. Human molecular genetics 18, 3110-3124 (2009).

29 Hoshi, M., Batourina, E., Mendelsohn, C. \& Jain, S. Novel mechanisms of early upper and lower urinary tract patterning regulated by RetY1015 docking tyrosine in mice. Development 139, 2405-2415 (2012).

30 Madisen, L. et al. A robust and high-throughput Cre reporting and characterization system for the whole mouse brain. Nature neuroscience 13, 133-140 (2010).

31 Chomez, P. et al. Increased cell death and delayed development in the cerebellum of mice lacking the rev-erbA(alpha) orphan receptor. Development 127, 1489-1498 (2000).

32 Storch, K. F. et al. Intrinsic circadian clock of the mammalian retina: importance for retinal processing of visual information. Cell 130, 730-741 (2007).

33 Mittelstadt, P. R., Monteiro, J. P. \& Ashwell, J. D. Thymocyte responsiveness to endogenous glucocorticoids is required for immunological fitness. The Journal of clinical investigation 122, 2384-2394 (2012).

34 Hinoi, E. et al. The sympathetic tone mediates leptin's inhibition of insulin secretion by modulating osteocalcin bioactivity. J Cell Biol 183, 1235-1242 (2008).

35 Fonseca-Pereira, D. et al. The neurotrophic factor receptor RET drives haematopoietic stem cell survival and function. Nature 514, 98-101 (2014).

36 Wiles, S., Pickard, K. M., Peng, K., MacDonald, T. T. \& Frankel, G. In vivo bioluminescence imaging of the murine pathogen Citrobacter rodentium. Infect Immun 74, 5391-5396 (2006). 
37 Collins, J. W. et al. Citrobacter rodentium: infection, inflammation and the microbiota. Nat Rev Microbiol 12, 612-623 (2014).

38 Rakoff-Nahoum, S., Paglino, J., Eslami-Varzaneh, F., Edberg, S. \& Medzhitov, R. Recognition of commensal microflora by toll-like receptors is required for intestinal homeostasis. Cell 118, 229-241 (2004).

39 Druzd, D. et al. Lymphocyte Circadian Clocks Control Lymph Node Trafficking and Adaptive Immune Responses. Immunity 46, 120-132 (2017).

40 Henslee, E. A. et al. Rhythmic potassium transport regulates the circadian clock in human red blood cells. Nat Commun 8, 1978 (2017).

41 Refinetti, R., Lissen, G. C. \& Halberg, F. Procedures for numerical analysis of circadian rhythms. Biol Rhythm Res 38, 275-325 (2007).

42 Moolenbeek, C. \& Ruitenberg, E. J. The Swiss Roll - a Simple Technique for Histological Studies of the Rodent Intestine. Lab Anim 15, 57-59 (1981).

43 Burich, A. et al. Helicobacter-induced inflammatory bowel disease in IL-10- and T cell-deficient mice. Am J Physiol Gastrointest Liver Physiol 281, G764-778 (2001).

44 Fort, M. M. et al. A synthetic TLR4 antagonist has anti-inflammatory effects in two murine models of inflammatory bowel disease. J Immunol 174, 6416-6423 (2005).

45 Seamons, A., Treuting, P. M., Brabb, T. \& Maggio-Price, L. Characterization of dextran sodium sulfate-induced inflammation and colonic tumorigenesis in Smad3(-/-) mice with dysregulated TGFbeta. PLoS One 8, e79182 (2013).

46 Veiga-Fernandes, $\mathrm{H}$. et al. Tyrosine kinase receptor RET is a key regulator of Peyer's Patch organogenesis. Nature 446, 547-551 (2007).

47 Tapial, J. et al. An atlas of alternative splicing profiles and functional associations reveals new regulatory programs and genes that simultaneously express multiple major isoforms. Genome Res 27, 1759-1768 (2017).

48 Law, C. W., Chen, Y., Shi, W. \& Smyth, G. K. voom: Precision weights unlock linear model analysis tools for RNA-seq read counts. Genome Biol 15, R29 (2014).

49 Huber, W. et al. Orchestrating high-throughput genomic analysis with Bioconductor. Nature methods 12, 115-121 (2015).

50 Ritchie, M. E. et al. limma powers differential expression analyses for RNAsequencing and microarray studies. Nucleic Acids Res 43, e47 (2015).

51 Cornelissen, G. Cosinor-based rhythmometry. Theor Biol Med Model 11, 16 (2014). 
Extended Data Figure 1. Clock genes in progenitors and gut ILC. a, Circadian clock gene expression in bone marrow innate lymphoid cell type 2 progenitor (ILC2P), small intestinal group 1 and 2 innate lymphoid cells (ILC1 and ILC2). ILC2P n=4; ILC1 n=3; ILC2 $n=6 . \mathbf{b}$, Per1 ${ }^{\text {Venus }}$ MFI analysis in bone marrow innate lymphoid cell type 2 progenitor (ILC2P) and small intestine lamina propria ILC1 and ILC2. ILC2P n=6; ILC1 and ILC2 $n=4$. c, Circadian clock gene expression in bone marrow common lymphoid progenitor (CLP), innate lymphoid cell progenitors (ILCP) and innate lymphoid cell type 2 progenitor (ILC2P). $n=3$. d, Circadian clock gene expression in small intestine lamina propria group 1 and 2 innate lymphoid cells (ILC1 and ILC2). ILC1 $n=3$; ILC2 $n=6$. (b-d) White: light period; Grey: dark period. Data are representative of 3 independent experiments. (a,c,d) $n$ represents biologically independent samples. (b) $n$ represents biologically independent animals. Mean and error bars: s.e.m. (a) two-way ANOVA followed by Tukey's multiple comparison test. $\mathrm{P}$ values are relative to differences for Per1 expression in ILC1 and ILC2 when compared with ILC2P; (b,d) Cosinor regression was used to define circadian rhythmicity; ${ }^{*} \mathrm{P}<0.05$; ${ }^{* \star} \mathrm{P}<0.01$; ${ }^{* *} \mathrm{P}<0.001$; ns not significant.

Extended Data Figure 2. Circadian signals regulate gut ILC3. a, Percentage of lamina propria group 3 innate lymphoid cells (ILC3); CD4 ${ }^{-} \mathrm{NCR}^{-}$, $\mathrm{LTi} \mathrm{CD4}^{+}$, and $\mathrm{NCR}^{+}$ ILC3 subsets. $n=6$. $\mathbf{b}$, Cell numbers of lamina propria group 3 innate lymphoid cells CD4 ${ }^{-N C R}{ }^{-}, \mathrm{LTi} \mathrm{CD}^{+}$, and NCR ${ }^{+}$ILC3 subsets. $\mathrm{n}=6$. c, Percentage of donor cells in group 2 innate lymphoid cells (ILC2), CD4 ${ }^{+} \mathrm{T}$ cells and Th17 subsets of mixed bone

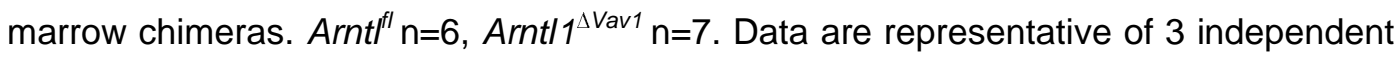
experiments. (a-c) $n$ represents biologically independent animals. Mean and error bars: s.e.m.. (a-c) two-tailed Mann-Whitney $U$ test. ${ }^{* \star} \mathrm{P}<0.01$; ns not significant.

\section{Extended Data Figure 3. Cell-intrinsic Arntl signals control intestinal ILC3. a,} Representative histogram of RFP expression in small intestine lamina propria ILC3. Representative of 3 independent analysis. b, Number of Peyer patches. Arnt $f^{f l}$ and 
$\operatorname{Arnt}^{\wedge \operatorname{Vav} 1} \mathrm{n}=9 ; \operatorname{Arnt}^{f l}$ and $\operatorname{Arnt}^{\wedge \text { Rorgt }} \mathrm{n}=7$. c, Percentage of small intestine lamina propria ILC3. $n=5$. d, Percentage of CCR6 ${ }^{-} C^{-}, \mathrm{CCR}^{+}$(LTi-like), and NCR ${ }^{+}$ILC3 subsets. $\mathrm{n}=5$. e, Percentage of intestinal lamina propria CD4 ${ }^{+} \mathrm{T}$ cells and helper (Th) 17 cells. $\mathrm{n}=5$. $\mathbf{f}$, Percentage and cell numbers of ILC3, regulatory T cells (Tregs) and RORyt expressing Tregs in the colonic lamina propria. $n=6$. $\mathbf{g}$, Percentage of lamina propria ILC3, CCR6 NCR ${ }^{-}$CCR6 $^{+}$(LTi-like), and NCR ${ }^{+}$ILC3 subsets. $n=3$. h, Percentage of donor cells and cells numbers of intestinal ILC3 in mixed bone marrow chimeras. $n=4$. i, Percentage of donor cells in CCR6 ${ }^{-} \mathrm{NCR}^{-}, \mathrm{CCR}^{+}$(LTi-like), and NCR ${ }^{+}$ILC3 subsets of mixed bone marrow chimeras. $\mathrm{n}=4$. j, Faecal Bacteroidetes and Firmicutes. Arnt $\left.\right|^{f t}$

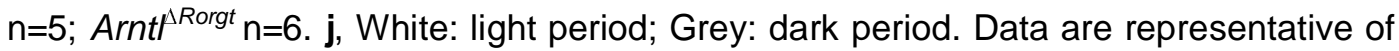
at least 3 independent experiments. (a-j) $n$ represents biologically independent animals. Mean and error bars: s.e.m.. (b-f,h-i) two-tailed Mann-Whitney U test; (j) Cosinor regression was used to define circadian rhythmicity; (g) two-tailed unpaired Student t-test. ${ }^{*} \mathrm{P}<0.05 ;{ }^{* \star} \mathrm{P}<0.01 ;{ }^{* \star *} \mathrm{P}<0.001$; ns not significant.

Extended Data Figure 4. Impact of Nr1d1 in intestinal ILC3. a, Percentage and cell numbers of small intestine lamina propria ILC3. $N 1 d 1^{+/+} n=6 ; N 1 d 1^{-/-} n=5$. b. Percentage

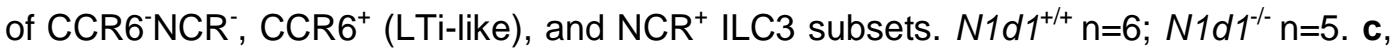
Schematic representation of mixed bone marrow chimeras setting. d, Percentage and cell numbers of donor cells in mixed bone marrow chimeras. $n=5$. e, Percentage of donor cells in intestinal CCR6 NCR, $\mathrm{CCR}^{+}$(LTi-like), and $\mathrm{NCR}^{+}$ILC3 subsets of mixed bone marrow chimeras. $n=5$. Data are representative of at least 3 independent experiments. (a-e) $\mathrm{n}$ represents biologically independent animals. Mean and error bars: s.e.m.. (a,b,d,e) two-tailed Mann-Whitney U test; ${ }^{* *} \mathrm{P}<0.01$; ns not significant.

\section{Extended Data Figure 5. ILC3-autonomous ablation of Arnt/ impairs intestinal} defence. a, b, Histopathology of colon and cecum in $\operatorname{Rag} 1^{1^{-/}}$. Arnt ${ }^{A \text { Rorgt }}$ and Rag $1^{-1-}$ .Arnt $f^{f l}$ littermate controls infected with $C$. rodentium. Pathological changes in colon and cecum of $\operatorname{Rag}^{1^{-1}}$. Arnt $\left.\right|^{\text {Rorgt }}$ mice included ulceration, loss of crypts and goblet cells, and 
inflammatory cell infiltration of the lamina propria by a granulocyte-rich population with a prominent and oedematous submucosa. Original magnification $\times 4$ (colon, scale bars: $500 \mu \mathrm{m}$ ); $\times 1.25$ (cecum, scale bars $250 \mu \mathrm{m}$ ). c, Inflammation score in the cecum. $\mathrm{n}=5 . \mathbf{d}$, Numbers of total ILC3; IL-17- and IL-22-producing ILC3. $n=3$. e, Whole-body imaging

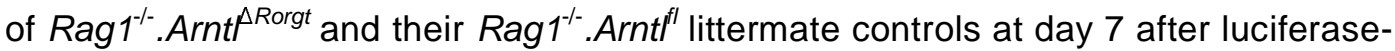
expressing $C$. rodentium infection. $\mathbf{f}$, MacConkey plates of liver cell suspensions

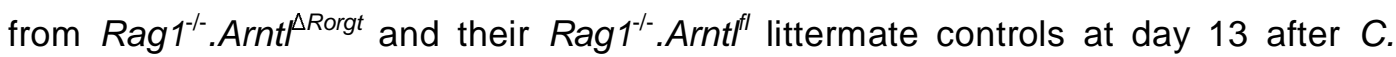
rodentium infection. $\mathbf{g}, \mathbf{h}$, Total bacteria (left) and $C$. rodentium (right) translocation to the liver and mesenteric lymph nodes $(m L N) . n=4$. i, Epithelial reactivity gene expression in the colon of C. rodentium infected $\operatorname{Rag}^{1^{-/}}$. Arnt $\left.\right|^{\Delta \text { Rorgt }}$ and their Rag $1^{-/-}$ Arnt $f^{f l}$ littermate controls. $\mathrm{n}=3$. j, Weight loss in C. rodentium-infected mice. Rag $1^{-/-}$ Arnt $^{f l} \mathrm{n}=6 ; \operatorname{Rag}^{-/}$. Arnt ${ }^{\text {Rorgt }} \mathrm{n}=7$. k, I, m, Histopatholgy analysis of inflammatory infiltrates in the liver, gonadal and subcutaneous fat. Liver, scale bars: $250 \mu \mathrm{m}$; gonadal and subcutaneous fat, scale bars: $100 \mu m n=4$. $n$, mouse total body weight. $n=5$. Data are representative of at least 3 independent experiments. $(a-n) n$ represents biologically independent animals. Mean and error bars: s.e.m.. (c,g,h) two-tailed MannWhitney $U$ test. $(d, n)$ two-tailed unpaired Student t-test. (j) two-way ANOVA and Sidak's test. ${ }^{*} \mathrm{P}<0.05 ;{ }^{* *} \mathrm{P}<0.01 ;{ }^{* * *} \mathrm{P}<0.001$; ns not significant.

Extended Data Figure 6. ILC3 proliferation, apoptosis and gut homing markers. a, ILC3-related gene rhythmicity in the small intestinal lamina propria ILC3. $n=4$. b, RNAseq analysis of lamina propria ILC3 at ZT23. n=3. c, Percentage of Ki67 expression in small intestine lamina propria ILC3. $n=4$. d, Percentage of donor cells in mixed bone marrow chimeras. $n=4$. e, Number of Lin $^{-}$CD $127^{+}{ }^{R O R y t^{+}}$cells in the blood. $n=4$. $f$, Percentage of ILC3 in mesenteric lymph nodes (mLN). Arntt ${ }^{f l} \mathrm{n}=6 ; \operatorname{Arnt}^{\wedge \text { Rorgt }} \mathrm{n}=8 . \mathbf{g}$, Diurnal expression of Ccr9 transcripts in gut ILC3. $n=4 . \mathbf{h}, \mathbf{i}$, Percentage of CCR9 expression in small intestinal lamina propria CCR6 ${ }^{-N C R}{ }^{-}, \mathrm{CCR}^{+}\left(\mathrm{LTi}-\right.$ like), and $\mathrm{NCR}^{+}$ ILC3 subsets, ILC2 and CD4 ${ }^{+}$T cells, $n=3$. j, Percentage of $\alpha 4 \beta 7$ expression in small 
intestine ILC1, ILC2 and CD4 ${ }^{+}$T cells, $n=4$. k, Percentage of CCR9 expression in gut ILC1, ILC2 and CD4 ${ }^{+}$T cells, $n=4$. I, m, Diurnal analysis of $a 4 \beta 7$ and CXCR4 expression in small intestine ILC3. $n=4$. (a,e,g,I,m) White: light period; Grey: dark period. Mean and error bars: s.e.m.. (a,c-m) $n$ represents biologically independent animals. (b) n represents biologically independent samples. (a,g) two-way ANOVA; (c,d,h-k) two-tailed Mann-Whitney U test; $(e, I, m)$ Cosinor regression was used to define circadian rhythmicity. (f) two-tailed unpaired Student t-test. ${ }^{*} \mathrm{P}<0.05$; ${ }^{* *} \mathrm{P}<0.01$; ${ }^{* \star *} \mathrm{P}<0.001$; ns not significant.

\section{Extended Data Figure 7. Light entrains intestinal ILC3 circadian oscillations. a,} Inverted feeding regimens in constant darkness. Per1 ${ }^{\text {Venus }}$ expression in gut ILC3. $n=3$. b, Circadian clock gene expression in hepatocytes of Per1 ${ }^{\text {Venus }}$ mice in inverted feeding regimens. $n=3$. Acrophase mean and s.e.m: Arntl: control $0.4 \pm 0.5$, inverted $11.5 \pm 0.2$; Per2: control $15.2 \pm 0.6$, inverted $3.9 \pm 0.5 ;$ Nr1d1: control $7.1 \pm 0.6$; inverted $18.5 \pm$ 0.8. c, Opposing light-dark cycles. Per1 ${ }^{\text {Venus }}$ in gut CCR6 NCR', LTi CD4 ${ }^{+}$, and $\mathrm{NCR}^{+}$ ILC3 subsets. $\mathrm{n}=3$. Acrophase mean and s.e.m: CCR6 ${ }^{-N C R}$ : control $14.5 \pm 0.5$, inverted $2.5 \pm 0.5 ; \mathrm{LTi} C D^{+}:$control $14.5 \pm 0.6$, inverted $2.5 \pm 0.4 ; \mathrm{NCR}^{+}$: control $14.5 \pm$ 0.6 , inverted $2.5 \pm 0.4$. d, Per1 ${ }^{\text {Venus }} \mathrm{MFI}$ analysis of small intestine lamina propria ILC3 in mice maintained in constant darkness for 28 days. $n=3$. Data are representative of 3 independent experiments. (a-d) $\mathrm{n}$ represents biologically independent animals. Mean and error bars: s.e.m.. Cosinor regression. Standard errors with 95\% confidence limits for amplitude (Amp) and acrophase (Acro) were extracted from the model and compared using two-tailed Student t-test. ${ }^{* *} \mathrm{P}<0.01$; ${ }^{* \star *} \mathrm{P}<0.001$; ns not significant.

Extended Data Figure 8. SCN-ablation shapes intestinal ILC3. a, Circadian clock gene expression in small intestinal lamina propria ILC3. $n=2-3 . \mathbf{b}$, Magnetic resonance imaging of sham and SCN ablated (xSCN) Per1 $1^{\text {venus }}$ mice. Sagittal slices. White arrows: SCN ablation. c, Rhythms of animal locomotor activity. Total distance travelled in meters. d, Nissl staining of coronal brain sections. Top panel; scale bar: $1 \mathrm{~mm}$. 
Bottom panel; scale bar $250 \mu \mathrm{m}$. (a-d) $\mathrm{n}$ represents biologically independent animals. Error bars show s.e.m.. Cosinor regression was used to define circadian rhythmicity; Cosine fitted curves are shown; Standard errors with 95\% confidence limits for amplitude (Amp) and acrophase (Acro) were extracted from the model and compared using two-tailed Student t-test. * $\mathrm{P}<0.05$; ns not significant.

Extended Data Figure 9. Brain-tuned signals shape gut ILC3. a, b, Confocal image of coronal brain sections showing neurotrace and RFP expression in the SCN. Scale bar: $100 \mu \mathrm{m}$. Representative of 3 independent analyses. c, Representative histogram of RFP expression in small intestine lamina propria ILC3. Representative of 3 independent analyses. d, Per1 expression in small intestinal lamina propria ILC3. $n=3$. e, Percentage of small intestine lamina propria ILC3. $n=3$. $\mathbf{f}$, Number of small intestine lamina propria ILC3. $n=3 . \mathbf{g}, \mathbf{h}$, Epithelial reactivity gene expression in the intestinal epithelium. $n=3$. i, Rhythms of faecal Bacteroidetes and Firmicutes. Arnt $f^{f l} n=4$,

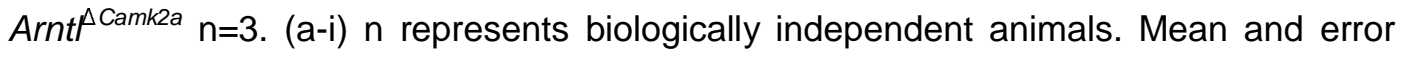
bars: s.e.m.. (d,g-i) Cosinor regression was used to define circadian rhythmicity; Cosine fitted curves are shown. (e) two-way ANOVA and Sidak's test; (f) two-tailed unpaired Student t-test; ${ }^{*} \mathrm{P}<0.05 ;{ }^{*} \mathrm{P}<0.0 ; 1$; ns not significant.

Extended Data Figure 10. Impact of Nr3c1 and Adrb2 deficiency in gut ILC3. a, Per1 ${ }^{\text {Venus }} \mathrm{MFI}$ analysis of lamina propria ILC3 after dexamethasone administration. $\mathrm{n}=$ 3. b, Percentage and cell numbers of small intestine ILC3. $n=3$. c, Percentage of lamina propria $\mathrm{CCR}^{-} \mathrm{NCR}^{-}, \mathrm{CCR}^{+}$(LTi-like), and $\mathrm{NCR}^{+}$ILC3 subsets. $\mathrm{n}=3$. d, Tyrosine hydroxylase (TH) expressing neurons (red) and RET positive ILC3 (green) in cryptopatches. Scale bars: $40 \mu \mathrm{m}$. Representative of 3 independent analyses. e, Normalized expression of Adrb1, Adrb2 and Adrb3 in CCR6 ${ }^{-N C R}$, $\mathrm{CCR}^{+}$, and NCR ${ }^{+}$ ILC3 subsets. f, Percentage and cell numbers of gut ILC3 in Adrb2 $2^{\triangle 1 / 7 r a}$ mice and their littermate controls. $n=6$. g, Percentage of lamina propria CCR6 ${ }^{-N C R}{ }^{-}, \mathrm{CCR}^{+}$(LTi-like), and $\mathrm{NCR}^{+}$ILC3 subsets in Adrb2 ${ }^{\Delta / 17 r a}$ mice and their littermate controls. $\mathrm{n}=6 . \mathbf{h}$, 
Percentage and cell numbers of gut ILC3 in Adrb2 $2^{\triangle \text { Rorgt }}$ mice and their littermate controls. $\operatorname{Adrb} 2^{\mathrm{fl}} \mathrm{n}=3 ; \operatorname{Adrb}^{\triangle \text { Rorgt }} \mathrm{n}=4$. i, Percentage of lamina propria CCR6 ${ }^{-} \mathrm{NCR}^{-}$, $\mathrm{CCR6}^{+}$(LTi-like), and NCR ${ }^{+}$ILC3 subsets in Adrb2 $2^{\triangle \text { Rorgt }}$ mice and their littermate controls. $\operatorname{Adrb}^{\mathrm{fl}} \mathrm{n}=3 ; \operatorname{Adrb}^{\triangle R \operatorname{Rorgt}} \mathrm{n}=4$. j, light cues and brain-tuned circuits shape gut ILC3 homeostasis. Arrhythmic ILC3 impact intestinal homeostasis, epithelial reactivity, microbiota, enteric defence, and the host lipid metabolism. Thus, ILC3 integrate local and systemic entraining cues in a distinct hierarchic manner, establishing an organismal circuitry that is an essential link between diurnal light signals, brain cues, intestinal ILC3 and host homeostasis. (a-d,f-i) n represents biologically independent animals. (a) White: light period; Grey: dark period. Mean and error bars: s.e.m.. (a) twoway ANOVA and Sidak's test. (b,c,f-i) two-tailed Mann-Whitney $U$ test. ${ }^{*} P<0.05$; ${ }^{* * *} \mathrm{P}<0.001$; ns not significant. 

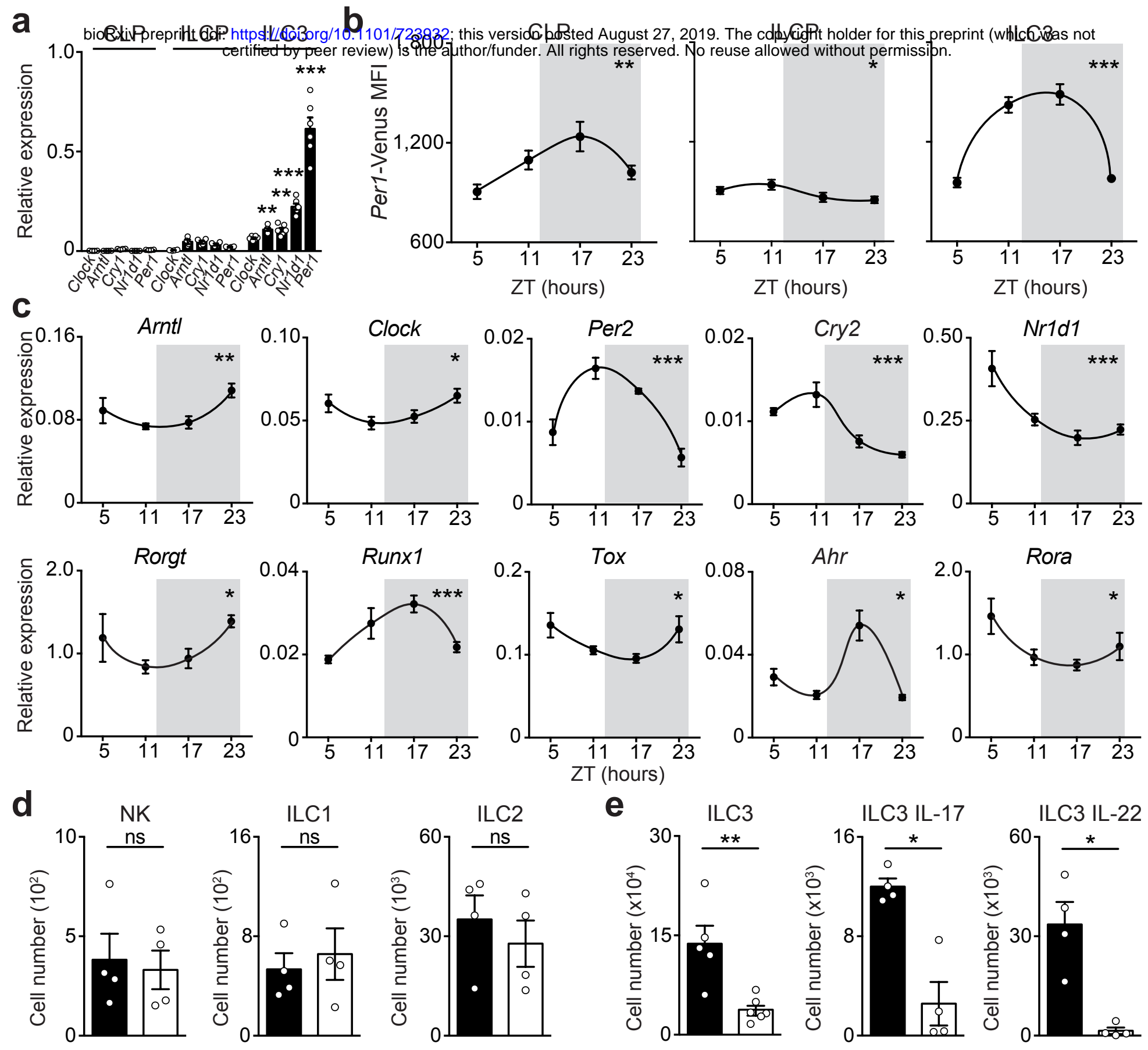

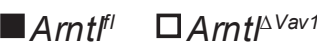

$\square A m t f^{f l} \quad \square A m t^{\Delta \operatorname{Vav} 1}$

$f$
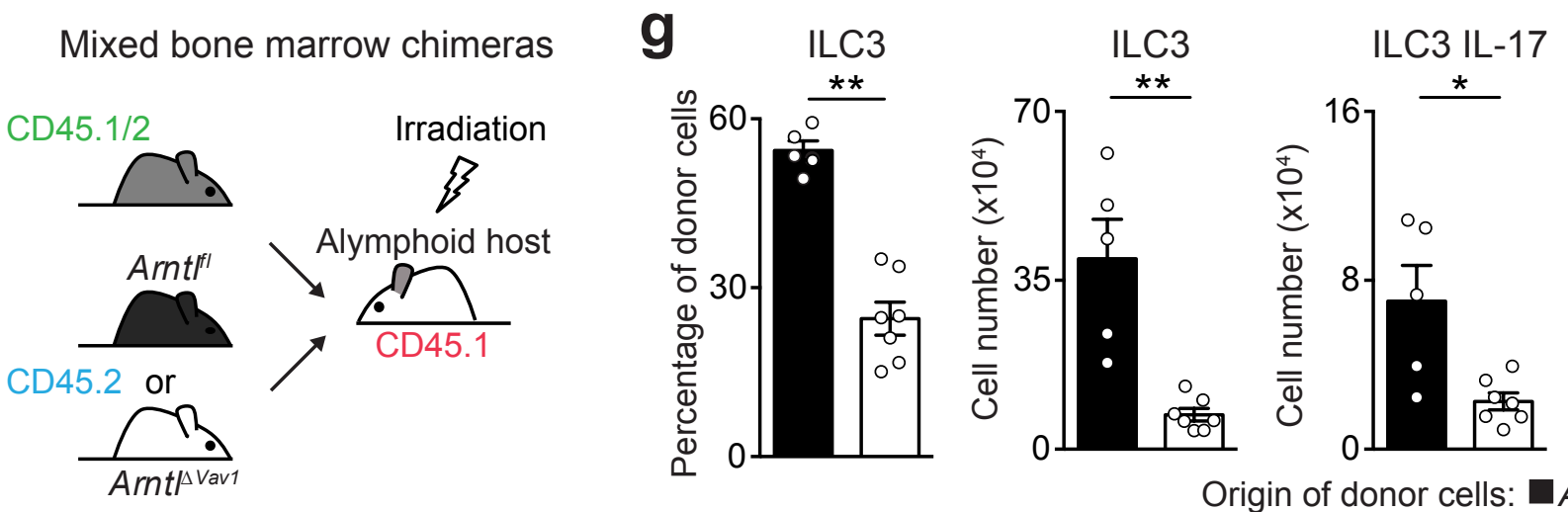

ILC3 IL-22

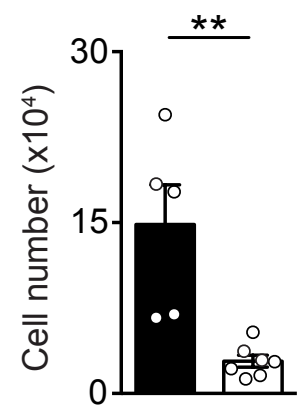

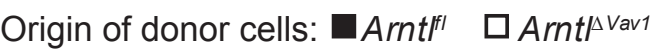


bioRxiv preprint doi: https://doi.org/10.1101/723932; this version posted August 27, 2019. The copyright 1 Ader for this preprint (which was not

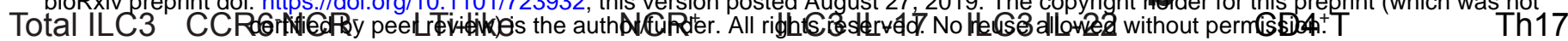
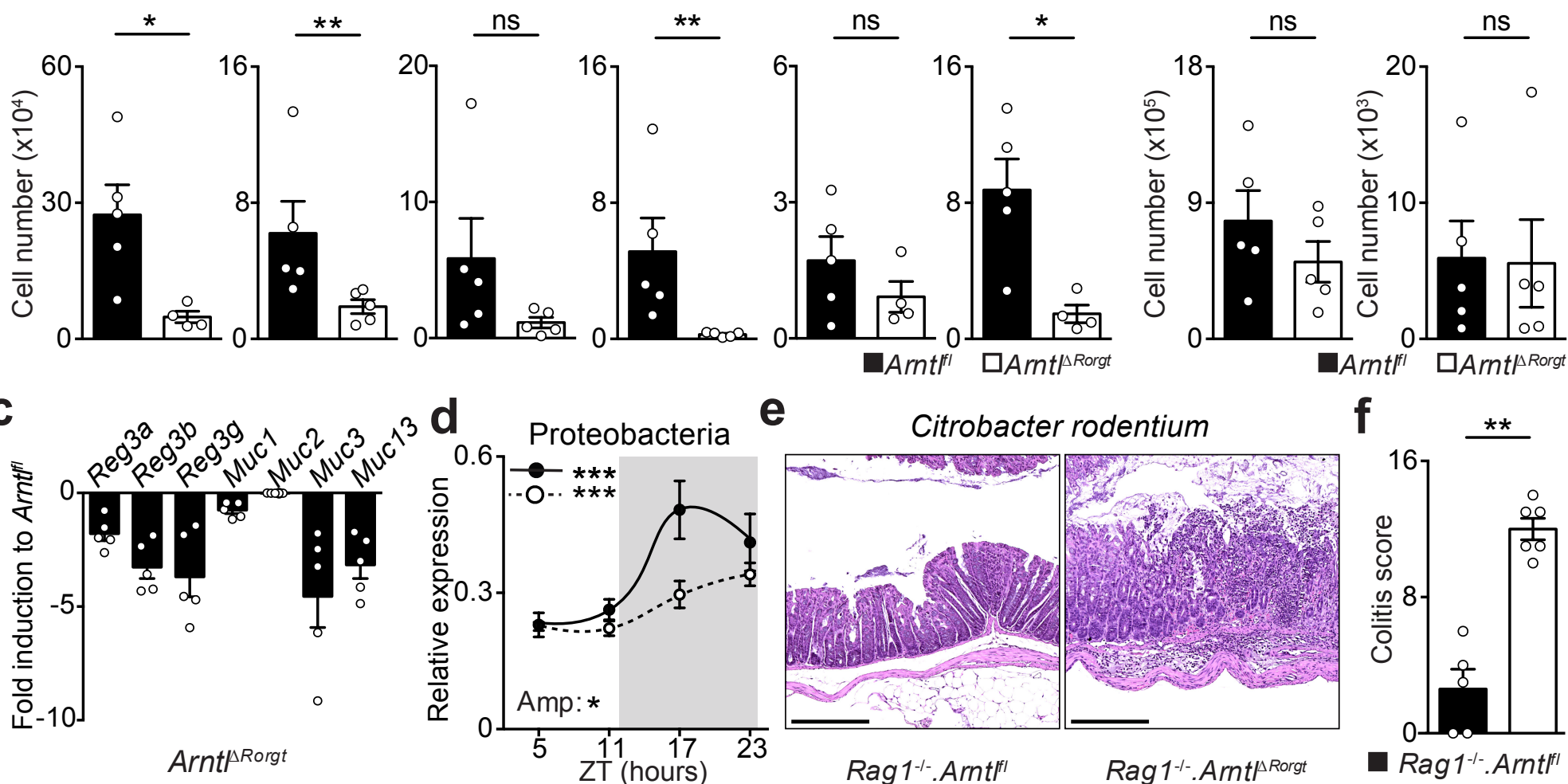

$\operatorname{Arnt}^{\wedge \text { Rorgt }}$

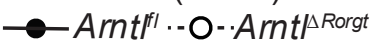

e

Citrobacter rodentium

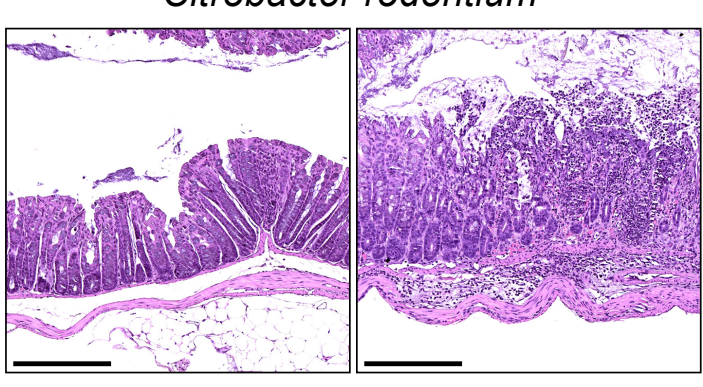

f

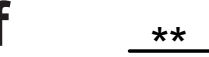

9

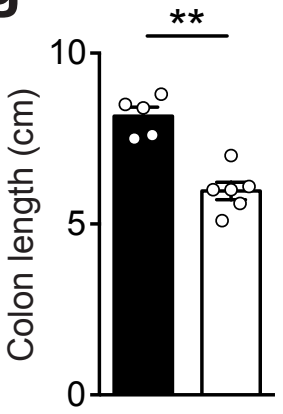

- $\operatorname{Rag} 1^{-1} \cdot$ Arnt $^{f t}$

$\square \operatorname{Rag}^{1 /-}$. Arnt $\left.\right|^{\text {Rorgt }}$ h

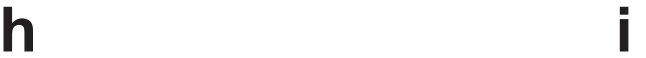

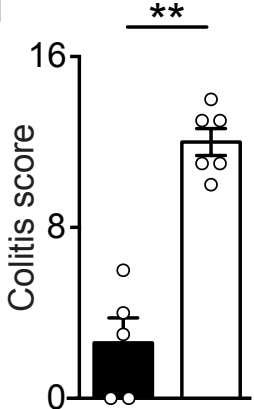

$\operatorname{Rag}^{1-\text { - }}$.Arnt $\left.\right|^{\text {Rorgt }}$
Rag $1^{-/}$.Arnt fl

$\square \operatorname{Rag}^{1-\text { - }}$.Arnt/ ${ }^{\text {Rorgt }}$

Bacteria C.rodentium j

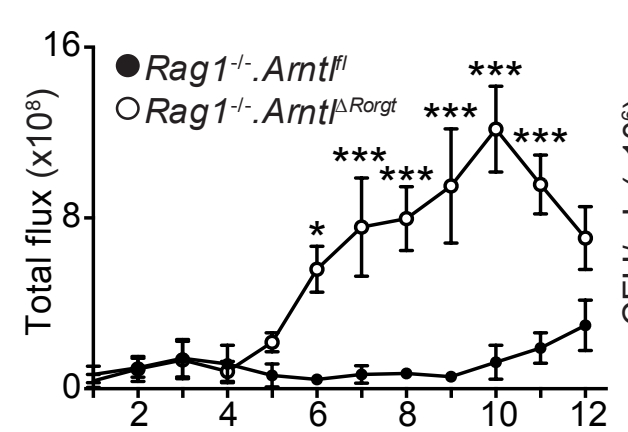

Day post-infection

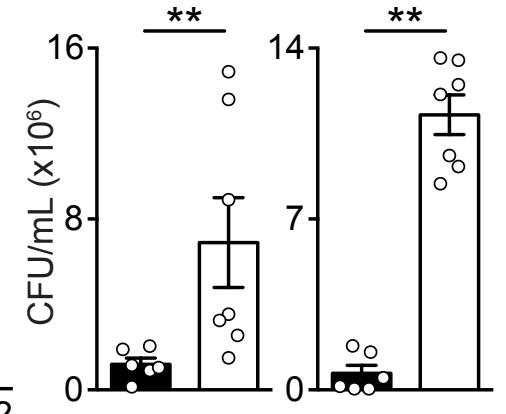

Rag $1^{-1-} \cdot$ Arnt $t^{f l}$

$\square \operatorname{Rag}^{1 /-}$.Arnt/RRorgt

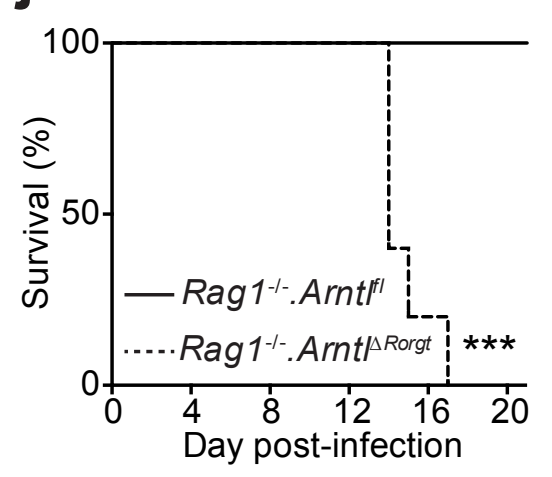

Fabp1

Fabp2

Scd1

Cd36

Apoe

I

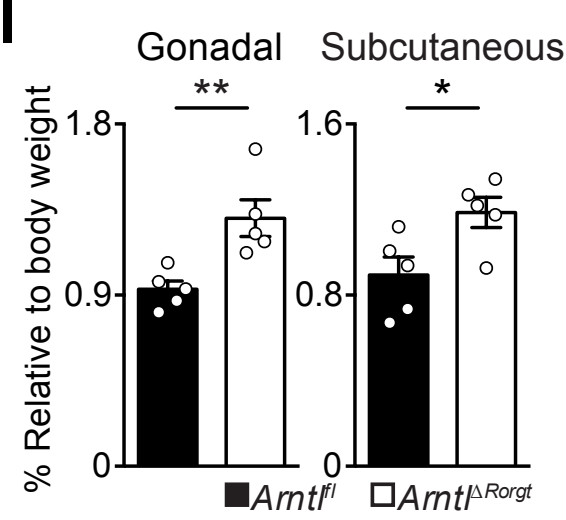


a
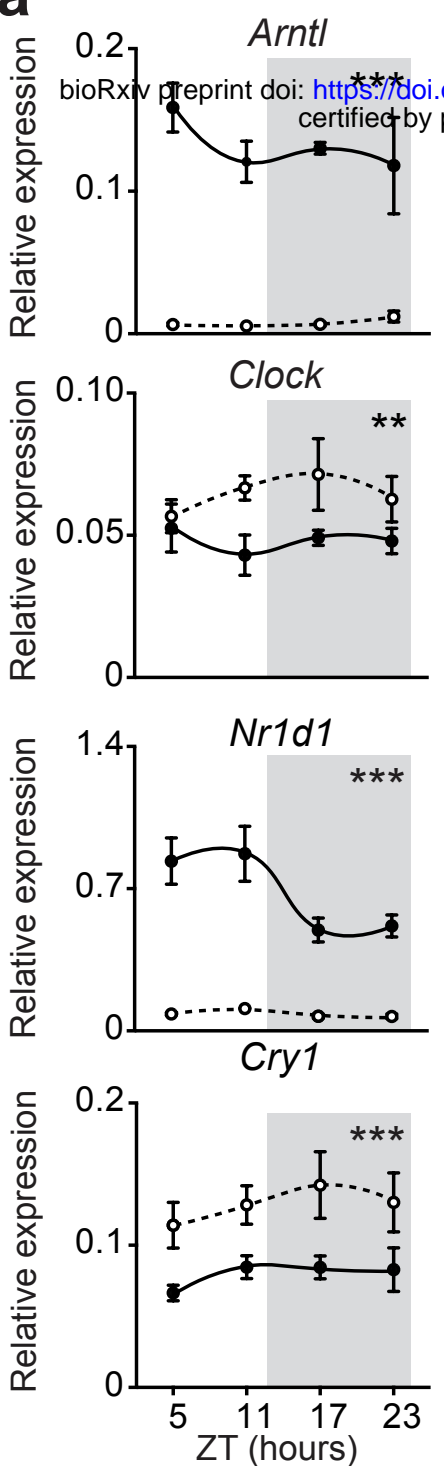

$\rightarrow A r n t f^{f l} \cdot \cdot \mathrm{O} \cdot \cdot \cdot$ Arnt $^{\Delta R o r g t}$ b

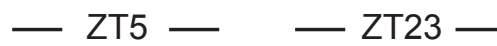

C

CLP

ILCP

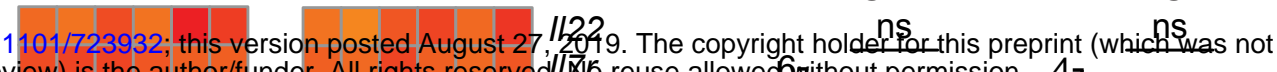
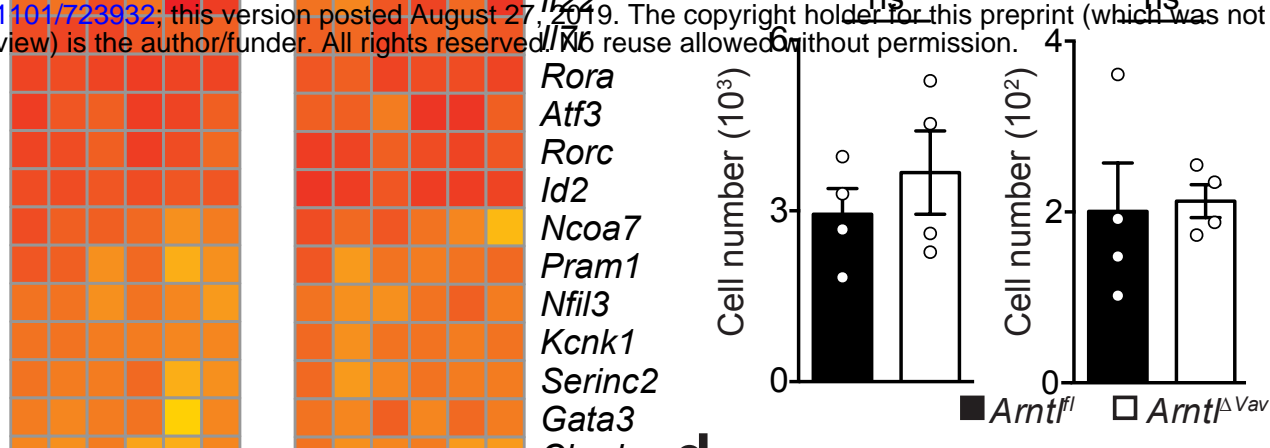

Gata3

d

Spleen ILC3

Tox

II23r

Lingo4

Scpep1

II1r1

Stat3

Smyd2

Thy1

Ccr6

Prelid2

Tifa

Cd93

Large

Lrrc49

Ifng

Zfp57

Tbx21

Ablim 3

Gpr157
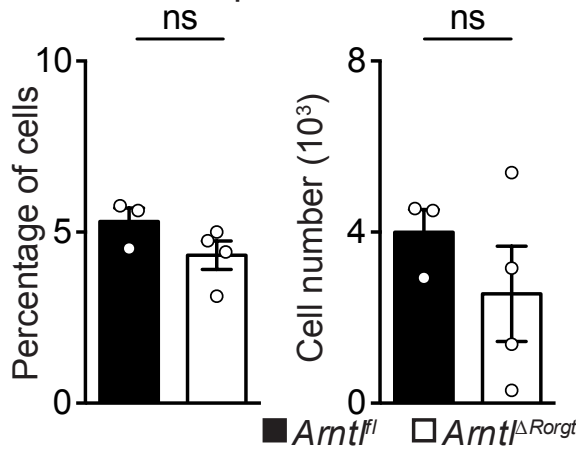

e

Lung ILC3
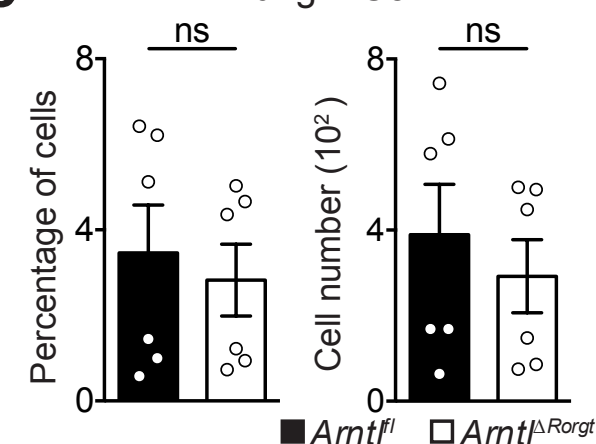

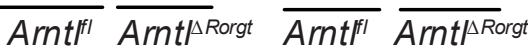

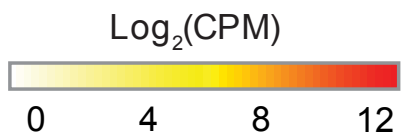

Asphd2

g

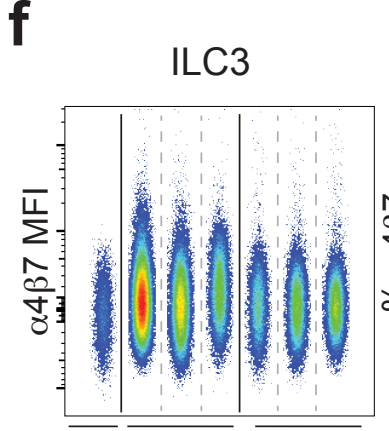

Iso Arntf Arnt $^{\Delta \text { Rorgt }}$

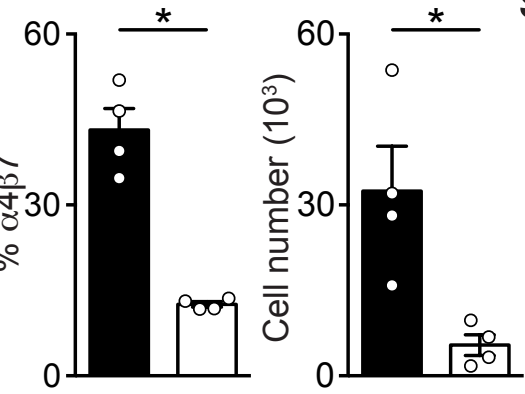

- Arntfil $\square$ Arnt/ ${ }^{\text {Rorgt }}$

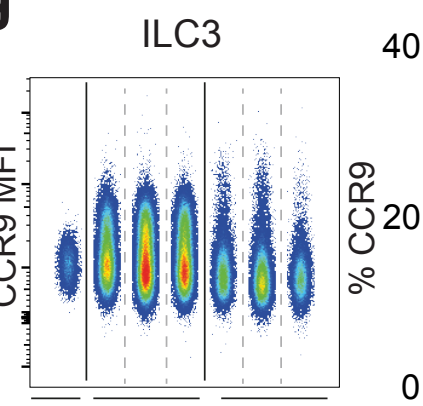

Iso Arntfl $\left.A r n t\right|^{\Delta R o r g t}$

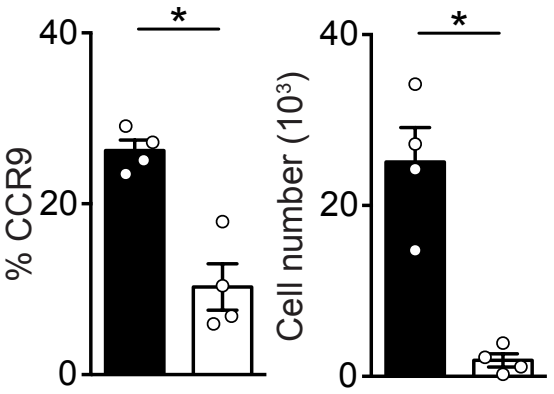

- Arntfl $\square$ Arnt/PRorg
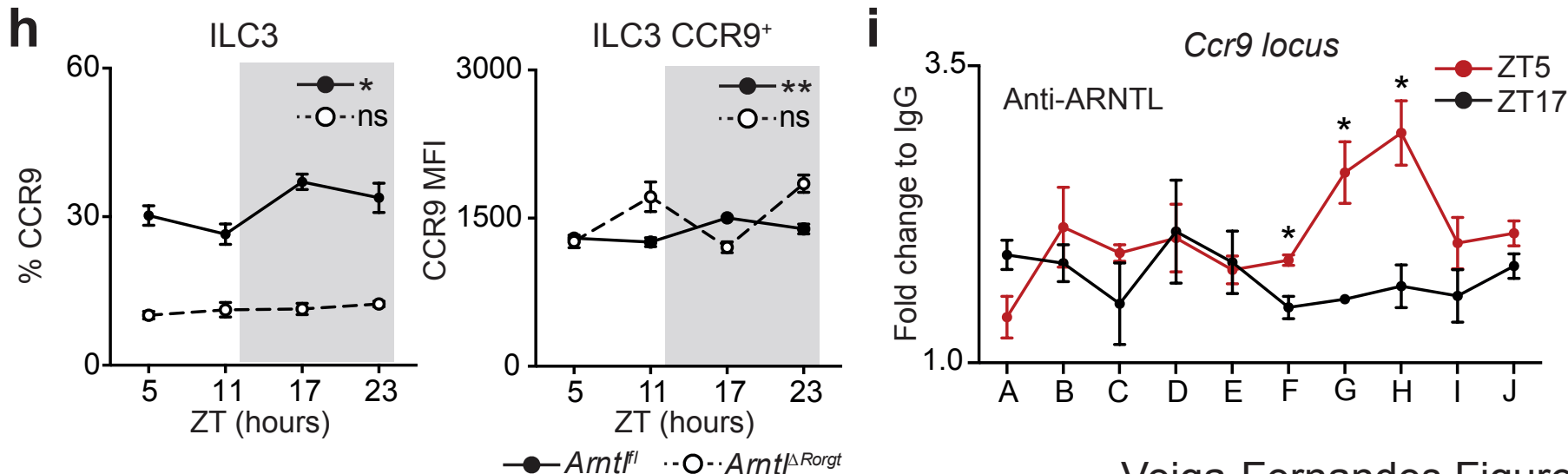

Veiga-Fernandes Figure 3 
C Light/Dark

$\left.{ }^{1,600}\right]$-Control ***

1,400

Control ***

1,600

Control $* * *$

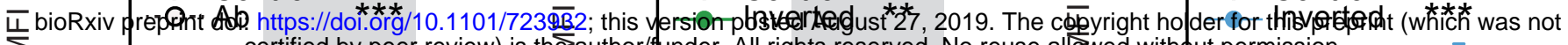
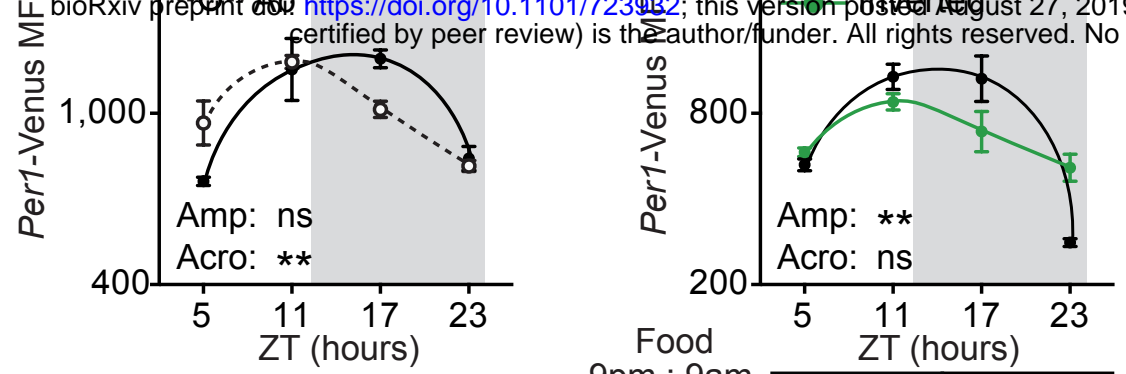

Food
$9 \mathrm{pm}: 9 \mathrm{am}$

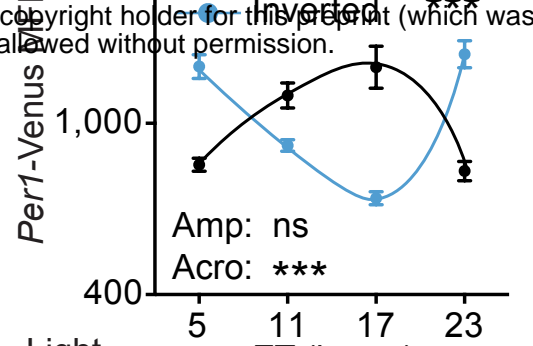

9am : 9pm

Light

9am : 9pm

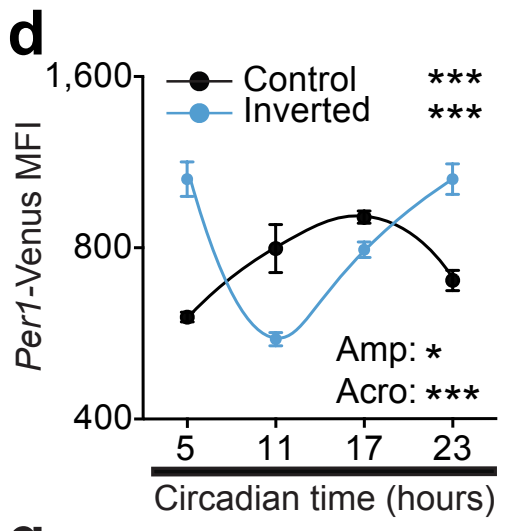

Fabp 1

.

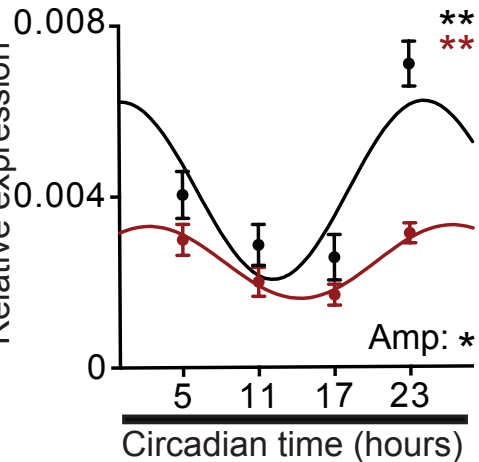

e

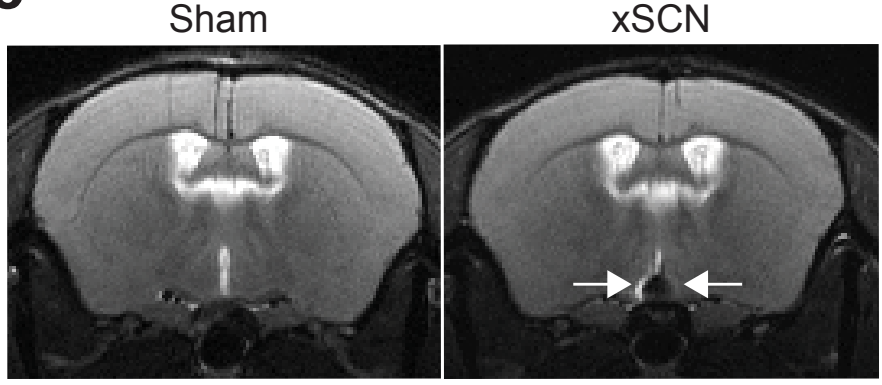

Nr1d1
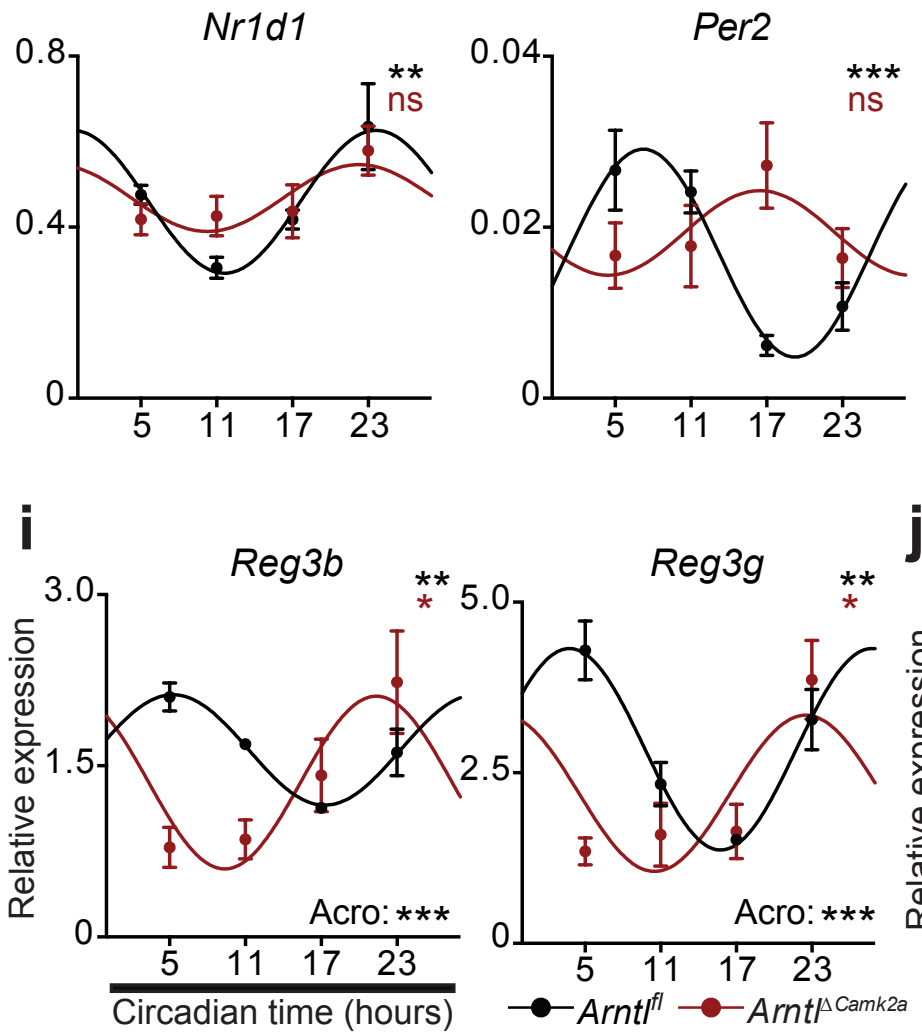

Fabp2

f

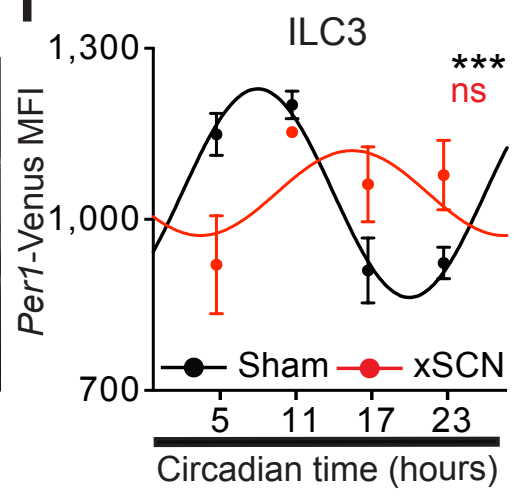

0.150

Cry1

*

ns

0.075

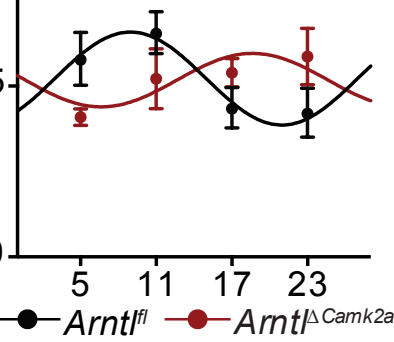

0.026 Proteobacteria

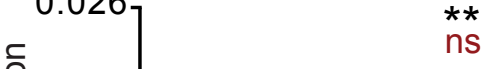

ns

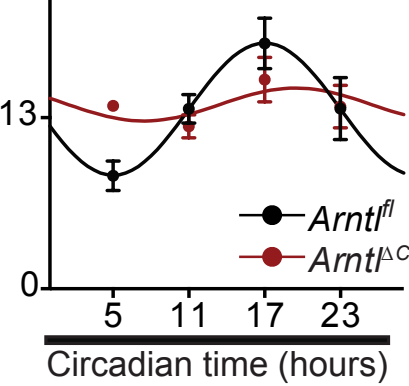

Gonadal Subcutaneous

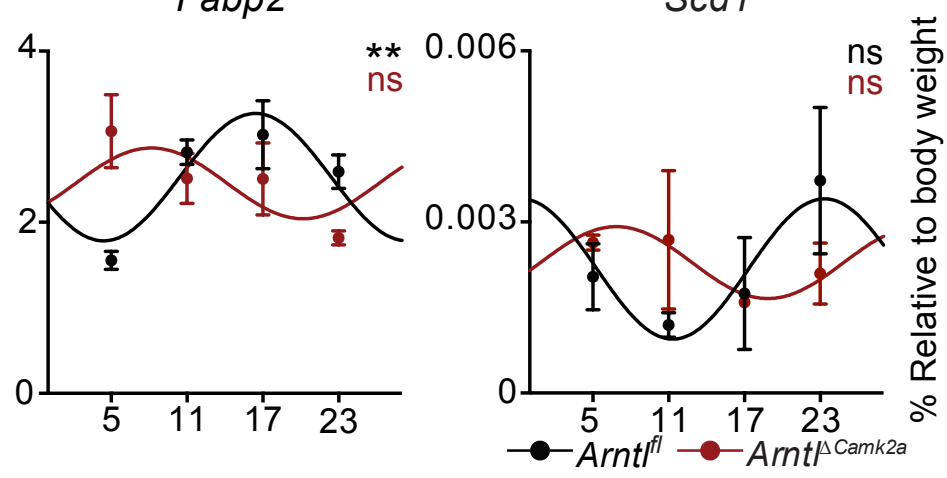

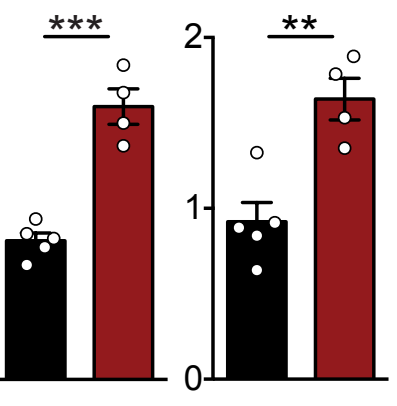

Arntf ${ }^{f l} \quad \square A r n t /$ Camk2a $^{2}$

Veiga-Fernandes Figure 4 